\title{
Bewertung unter Vorbehalt. Zur Praxis der Bewertungskommunikation in einem Streichquartett
}

\author{
Désirée Wilke
}

Online publiziert: 17. März 2017

(C) Der/die Autor(en) 2017. Dieser Artikel ist eine Open-Access-Publikation.

Zusammenfassung Der Beitrag untersucht einen besonderen Fall von interaktiver Bewertungspraxis anhand von Probengesprächen unter Musikern eines studentischen Streichquartetts. Für eine Soziologie der Bewertung ist diese Interaktionssituation ohne institutionalisierte Bewertungsinstanz - also ohne Dirigent oder Dirigentin - interessant, insofern das wechselseitige Bewerten von Interpretationsvorschlägen und Spieltechniken durch die Musizierenden geradezu konstitutiv ist für die Erarbeitung einer gemeinsamen Interpretation eines Musikstückes, negative Bewertungen die Kooperation aber potenziell gefährden. Das Erkenntnisinteresse der empirischen Studie richtet sich nicht auf die inhaltliche Dimension der Bewertungskriterien, sondern erforscht konversationsanalytisch den kommunikativen Vollzug der Bewertungspraxis. Die Ergebnisse der Sequenzanalysen weisen nicht nur auf eine Vermeidung von Kränkungen bei der Bewertungskommunikation unter den Musikern hin, sondern zeigen auch, wie der Diskussionsraum durch unverbindlich formulierte Bewertungen weiterhin geöffnet bleibt, sodass Bewertungen zu einem späteren Zeitpunkt revidiert werden können. Die Offenheit der Bewertungskommunikation fördert somit einerseits die Beibehaltung der Integration der Akteure im Kooperationszusammenhang, ist andererseits aber dem Fehlen einer semantischen Eindeutigkeit der Klangbeschreibungen durch die Musiker geschuldet, da die deutsche Sprache für präzise Klangbeschreibungen kaum Worte bereitstellt, sodass hierfür vielfach Metaphern und außersprachliche Ausdrucksformen (z. B. Vorsingen, Vorspielen, Gesten) herangezogen werden müssen.

D. Wilke $(\square)$

Paris Lodron Universität Salzburg,

Fachbereich Politikwissenschaft und Soziologie,

Abteilung Soziologie und Kulturwissenschaft,

Rudolfskai 42

5020 Salzburg, Österreich 
Schlüsselwörter Bewertung $\cdot$ Streichquartett $\cdot$ Interaktion $\cdot$ Ethnomethodologie Konversationsanalyse

\title{
Judgement under reserve. The practice of communicating judgements in a string quartet
}

\begin{abstract}
The paper examines a specific case of interactive practice of judging, analyzing the rehearsal talk amongst musicians of a student string quartet. This interaction lacks an institutionalized instance of judging - respectively a conductor - and is therefore an interesting setting for a sociology of judgement: for, as reciprocal judging activity is downright constitutive for developing an interpretation of a piece of music together, negative judgements can also endanger the cooperation between the musicians. The epistemological interest of this empirical study is not directed towards the content of the criteria of judgements. Instead, the study explores the communicative performance of judging by using conversation analysis. The results of the sequence analysis not only reveal that musicians avoid insults whilst making their judgements, but also that judgements are formulated tentatively for the discussions to remain more or less open-ended, as to facilitate the revising of judgements at a later point of time. On the one hand, this open-ended communication of judgements helps maintaining the integration of the musicians in the cooperation; on the other hand, open-ended communication also reflects the lack of semantic uniqueness when the musicians describe sound. For German language offers only few precise words for the description of sound, the use of metaphors and extralinguistic forms of expression (e.g. singing, musical performance, gesture) becomes necessary.
\end{abstract}

Keywords Judgement · String quartet · Interaction · Ethnomethodology · Conversation analysis

\section{Jugements sous réserve. Sur la pratique de la communication de jugements au sein d'un quatuor à cordes}

Résumé Cet article étudie un cas particulier de pratique interactive du jugement à partir de discussions menées par des étudiants membres d'un quatuor à corde lors de répétitions. Cette situation d'interaction dénuée d'un instance de jugement institutionnalisée, c'est-à-dire sans chef d'orchestre, est intéressante pour la sociologie du jugement dans la mesure où, si les jugements réciproques portés par les musiciens sur les propositions d'interprétation et de techniques de jeu est en quelque sorte constitutive de l'élaboration d'une interprétation commune d'un morceau de musique, les jugements négatifs compromettent potentiellement la coopération. L'intérêt de connaissance de cette étude empirique ne porte pas sur la teneur des critères de jugement mais sur la pratique du jugement en actes dans une perspective d'analyse de la conversation. L'analyse des séquences n'indique pas seulement le souci des musiciens de communiquer des jugements sans s'offenser. Elles montrent également comment l'espace de discussion est maintenu ouvert par des jugements de nature pro- 
visoire susceptibles d'être révisés ultérieurement. Ce mode ouvert de communication des jugements favorise, d'une part, le maintien des acteurs dans le contexte de coopération. Il est dû, d'autre part, à l'absence de sémantique univoque permettant aux musiciens de décrire les sons. La langue allemande dispose en effet de très peu de mots pour décrire précisément les sons, de telle sorte que les musiciens doivent souvent avoir recours à des métaphores ou des formes d'expression extralinguistiques (p. ex. chant, démonstration, gestes).

Mots-clés Jugement · Quatuor à cordes · Interaction · Ethnométhodologie · Analyse de conversation

\section{Einleitung}

Die nachfolgende Studie untersucht die Bewertungsinteraktion unter Musikern ${ }^{1}$ am Beispiel eines studentischen Streichquartetts. Die Musiker an erster und zweiter Violine, Viola und Violoncello müssen, um zu einer Interpretation zu gelangen, ihr jeweiliges Verstehen der kompositorischen Aussage und ihre individuellen künstlerischen Ausdrucksansprüche in einen gemeinsamen Klang überführen. Die im Zuge der musikalischen Sozialisation erworbenen Vorstellungen, Erfahrungen und Gewohnheiten im Umgang mit Musik geben ihnen hier Orientierung (vgl. Schütz 1972). Dennoch enthält ein Notentext aufgrund seiner Lücken (zur Lückenhaftigkeit von Notationen vgl. Adorno 2001, S. 11; Schütz 1972, S. 137) keine eindeutige Interpretationsanweisung. Das wechselseitige Kommunizieren von Bewertungen des Spiels ist daher konstitutiv für die Einigung auf eine Interpretation. Die Musiker können sich dabei nicht auf eine zentrale Bewertungsinstanz berufen, da ein Streichquartett keinen Dirigenten hat. Für eine Soziologie der Bewertung ist dieses Setting nicht nur aufgrund der dadurch gegebenen formalen Gleichberechtigung der Interaktionsteilnehmer interessant: Negative Bewertungen gefährden zudem potenziell die Kooperation. Im Hinblick auf die besonderen Rahmenbedingungen dieser Art von Interaktion untersucht die Studie daher nicht die Bewertungskriterien selbst, sondern den Einsatz kommunikativer Mittel im Zuge von Bewertungsprozessen. Zu diesem Zweck werden transkribierte Probengespräche eines studentischen Streichquartetts mithilfe der ethnomethodologischen Konversationsanalyse untersucht.

Zunächst wird der Arbeitskontext der Musiker beleuchtet, indem die institutionelle Sonderstellung des Streichquartetts erörtert wird. Danach folgt eine Bestandsaufnahme vorhandener Studien zu Probengesprächen im Streichquartett und in anderen kammermusikalischen Ensembleformen. Anschließend wird dargelegt, inwiefern eine ethnomethodologische Perspektive einen vertiefenden Einblick in die Praxis des Bewertungsvollzugs zwischen den Musikern ermöglicht. Die konversationsanalytisch ausgewerteten Forschungsergebnisse bereiten auf die abschließende Diskussion der Besonderheiten der Bewertungskommunikation in einem Streichquartett vor.

\footnotetext{
${ }^{1}$ Aus Gründen der besseren Lesbarkeit wird in der Studie durchgehend die maskuline Sprachform verwendet, womit immer beide Geschlechter gemeint sind.
} 


\section{Die institutionelle Sonderstellung des Streichquartetts}

Die gattungsgeschichtlichen Besonderheiten des Streichquartetts sind Teil der musikalischen Sozialisation und gehören, mit Alfred Schütz gesprochen, zum Korpus des für die musikalische Praxis handlungswirksamen „sozial abgeleiteten Wissens“ (1972, S. 139). Der folgende Abschnitt soll die Leser für diese Besonderheiten sensibilisieren.

Kaum eine Musikgattung kann auf eine so ausgeprägte Gattungstheorie zurückblicken wie das Streichquartett. Zu den zentralen Themen dieser Theorie gehören so zitiert Joachim Brügge (2004, S. 18) aus einem Lexikon-Eintrag in Die Musik in Geschichte und Gegenwart - die ,Würde“ und die „Diskursivität“ des Streichquartetts (Finscher 1965, Sp. 1925 ff.). Bei diesen beiden Charakterisierungen handelt es sich um außermusikalische Topoi, die mit innermusikalischen Qualitäten wie der Komplexität der Melodielinien, der Übergabe und Annahme von Melodie- und Begleitstimmen sowie den ausbalancierten Harmonien im vierstimmigen Satz verknüpft werden. Inwiefern die einzelnen Kompositionen diese Kriterien tatsächlich erfüllen, sodass man sie der Streichquartett-Theorie entsprechend als gattungstypische Kompositionen ansehen kann, ist eine musikwissenschaftliche Frage. Die mit der Rede von „Würde“ und „Diskursivität“ einhergehende Personifikation des Streichquartetts weckt hingegen das soziologische Interesse. Was hat es mit diesen sozial anmutenden Zuschreibungen auf sich?

Eine erste Antwort auf diese Frage bietet ein historischer Blick auf das Streichquartett als ein besonderer Typ von Kammermusik. Der Begriff „Kammermusik“ (abgeleitet vom italienischen „musica da camera“) hielt ab Mitte des 16. Jahrhunderts nach und nach Einzug in den musikalischen Sprachgebrauch. Nicola Vicentino war im Jahr 1555 einer der Ersten, die zwischen ,da cantare in chiesa“ (in der Kirche Gesungenes) und „da cantare in camera“ (im Zimmer Gesungenes) einen Unterschied machten (Vicentino 1555, lib. IV, cap. XIX, zitiert nach Salmen 1969, S. 10). Der Kammermusikbegriff verweist auf eine besondere soziale Realität. In Abgrenzung zur kirchlichen Musik sowie zur Oper oder zur Festmusik für ein breiteres Publikum zielt er auf eine Musikpraxis in privaten Räumen. Unter Kammermusik ,,verstand man [...] ursprünglich die , stillere “ Musik für elitäre Kreise in einer ständisch geschichteten Gesellschaft“" (Salmen 1969, S. 10), die sich vor allem in Deutschlands, Frankreichs und Italiens Adelshäusern großer Beliebtheit erfreute. Ausführende und Zuhörende sahen sich als Teil eines Expertenkreises für eine anspruchsvolle Kunst (ebd., S. 11). Vom ,elitären Kreis“ der Kammermusik lässt sich ein Bogen zur „Würde“ des Streichquartetts schlagen: Auch das Streichquartett gewinnt seine besondere Bedeutung aus der intimen und gehobenen Form der Musikpraxis, die für ein erlesenes Publikum von Kennern bestimmt ist.

Stilgeschichtlich durchlebte die Kammermusik mit der Abkehr vom Generalbass ${ }^{2}$ ab dem ersten Drittel des 18. Jahrhunderts eine Wandlung. Die musikalische For-

\footnotetext{
${ }^{2}$ Zur Zeit des Barock, ab 1600 bis ca. 1750, steht die Musik im Zeichen des Generalbasses. Er wird z.B. von Violoncello, Kontrabass, Orgel, Laute, Theorbe oder Cembalo gespielt. Seine Aufgabe ist es, „Fundament zu sein, akkordische Ausfüllung zu geben und das Ensemble beim Musizieren in Takt und Tempo zusammenzuhalten“ (Finscher 1974, S.107).
} 
menvielfalt nahm dadurch zu und führte zu mehr Gleichberechtigung zwischen den einzelnen Stimmen, was jenen Instrumenten, die bis dahin ausschließlich eine begleitende Funktion hatten, einen Zuwachs an spieltechnischen Möglichkeiten bescherte. Die Bassstimme entwickelte sich zu einer melodiehaften Stimme, die am Oberstimmen-,,Gespräch“ gleichberechtigt teilnehmen konnte (vgl. Finscher 1974, S. 107). Die Idee der Gleichberechtigung zwischen den Stimmen entfaltete sich in der Kammermusik zusehends und erfuhr im Zuge der Entstehung des Streichquartetts in der zweiten Hälfte des 18. Jahrhunderts - die somit ins fortgeschrittene Aufklärungszeitalter fällt - einen Höhepunkt. Die rationalistische Geisteshaltung dieser Zeit führte nicht nur zu Umbrüchen in Wirtschaft, Politik und Wissenschaft, sondern machte sich auch in einer veränderten Kunstauffassung bemerkbar. Vor diesem historischen Hintergrund sind die Zuschreibungen von ,Würde“ und „Diskursivität“ zu sehen. Die Rede von ,Würde“ transportiert das Bild der elitären Exklusivität der Streichquartettmusik ,im kleinen Kreis“", die noch auf die aristokratische Musikwelt verweist, wohingegen die Zuschreibung der ,Diskursivität“ das Bild der Gleichberechtigung von vier miteinander in einem egalitären Diskurs stehenden vernünftigen Stimmen vermittelt, das eher aufklärerisch anmutet. Wie sehr gerade der Topos der Diskursivität weiterhin aufgegriffen wird, wird durch die Tatsache belegt, dass in Feuilletons und Konzertbesprechungen auch heute noch gelegentlich auf ein Goethe-Zitat verwiesen wird, in dem die Diskurs- und Verstandesmetapher mit der Streichquartettmusik in Verbindung gebracht werden: „Dieser Art Exhibitionen [Quartettabende, D.W.] waren mir von jeher von der Instrumentalmusik das Verständlichste, man hört vier vernünftige Leute sich untereinander unterhalten, glaubt ihren Diskursen etwas abzugewinnen und die Eigentümlichkeiten der Instrumente kennenzulernen“ (Goethe 1976, S. 349).

Die Zuschreibungen „Würde“ und „Diskursivität“ sind einerseits Teil der musikwissenschaftlichen Sicht auf das Streichquartett, zeigen andererseits aber in ihrer anthropomorphisierenden Wortwahl auch, dass diese Sicht nicht allein rein sachlicher Natur ist. Sehr deutlich wird dies in folgendem Lexikon-Eintrag zum Streichquartett, der fast so alt ist wie die Gattung selbst. Im Universal-Lexicon der Tonkunst von 1837 heißt es: ,[Das Streichquartett] gleicht in diesem Betracht [...] einer beseelten Unterhaltung fühlender Menschen über die geheimsten Anliegen des Herzens, wobei die erste Violine als ein feurig schwärmerischer Jüngling gern das erste Wort führt, während der theilnehmende $\mathrm{Ba}$, ein harmonisch gebildeter Alter, das Gespräch nach den Gesetzen der Association fortführen und die Idee zusammen zu halten sich angelegen seyn lässt" (Fink 1837, S. 591). Diese vermenschlichende Beschreibung des Zusammenspiels im Streichquartett, die sich fernab von heute gängigen Formulierungen eines Lexikon-Artikels bewegt, gibt einen Hinweis darauf, dass die musikwissenschaftliche Streichquartett-Theorie von Beginn an auch von einer bis heute fortdauernden Mythosbildung um das Streichquartett inspiriert wurde, die die Lebenswelt von Streichquartett-Musikern in den Fokus rückt. Der Teil der Rezeptionsgeschichte des Streichquartetts, der hier als „Mythos“ bezeichnet wird, umfasst die vielen Geschichten, die sich um das Zusammenspiel und das Verhältnis der Musiker ranken, und setzt sich von einer musikologischen Betrachtung der Gattung ab. Zentrale Themen des Streichquartett-Mythos sind die Hierarchie zwischen den Musikern bzw. den Instrumenten, aber auch der Streit unter den Musikern. So greift die gelegentlich mit 
einem Augenzwinkern verwendete Formulierung von einer „Ehe zu viert“ (Simmenauer 2008) zwar die Idee der „Musik im kleinen Kreis“ auf, bezieht sich aber auf die partnerschaftliche Intimität und Nähe der Beziehungen der Musiker untereinander mit allen Vorzügen und Konflikten, die diese Beziehungskonstellation mit sich bringt. Im Streichquartett-Mythos wird zudem die Gleichberechtigungsidee oft zugunsten der Idee von festgelegten Rangordnungen unter den Instrumenten negiert. Beispiele hierfür finden sich bereits in der Alltagssprache. So spielt die Redewendung ,die erste“ bzw. „die zweite Geige spielen“ auf eine Hierarchie der Instrumente an, die zur Charakterisierung von Personen herangezogen wird.

Daneben findet sich eine ganze Reihe von literarischen Übersetzungen der Streichquartett-Praxis, die im Widerspruch zur Idee von den vier ,,vernünftigen“, gleichberechtigten Stimmen stehen. Sie imaginieren neben einer Hierarchie der Instrumente auch eine Festlegung jedes Instruments bzw. jedes Musikers, der das jeweilige Instrument spielt, auf klare und mitunter sogar geschlechtsstereotype Rollenmuster. So heißt es in dem Gedichtband Das Streichquartett von Alfred von Ehrmann (1903, S. 2 ff.):

\section{„Violino primo.}

O erste Geige, Königin!

Wahrhaftig, Kron' und Hermelin

Kommt dir von Gottes Gnaden zu,

Denn wo du bist, da herrschest du.

[...]

Beglückt, wer deiner Macht sich neige,

Glorreiche Herrin, erste Geige!

\section{Viola.}

Viola heißt die dritte Stimme, Sie schuf ein Gott in seinem Grimme!

[...]

Viola heißt: gebundne Kraft

Und heißt: verhaltne Leidenschaft.

\section{Violino secondo.}

Was soll die zweite Geige sein?

Der ersten holdes Schwesterlein,

[...]

Doch ohne allen Herrschergeist.

$\mathrm{Zu}$ dienen eher, sich zu fügen,

Der Aelteren sich anzuschmiegen,

Verlangt ihr stiller Mädchensinn:

Verborgen blühn dünkt ihr Gewinn.

\section{Violoncello.}

[...]

Doch nun zum erstenmale fällt

In den Diskant der Weibersippe

Ein kräftig Wort von bärt'ger Lippe,

Auf tut sich eine neue Welt:

Es öffnet sich, so groß und weit

Der Horizont der Männlichkeit.“

Darüber hinaus gibt es eine tradierte Scherzkultur, die den einzelnen Instrumenten und ihren Spielern ebenfalls eine unterschiedliche Wertigkeit beimisst (vgl. Schütte 1991; Bäuerle 1997). In dem humorvollen Buch für „Freunde häuslicher Musik“ Das stillvergnügte Streichquartett (Aulich und Heimeran 1936) werden Schilderungen scheinbar typischer Verhaltensweisen der jeweiligen Instrumentalisten in einem hierarchischen Gefüge gegeben. Diese Verhaltensweisen schließen nicht selten Ressentiments einzelner Musiker den anderen Ensemblemitgliedern gegenüber ein. Auch 
das szenische Drama Die Beleidigten von Ulrich Hub (1997) persifliert die Thematik der im Streichquartett aufkeimenden Neigung zum Streit. Dass in diesen Beispielen die Gleichberechtigungsidee konterkariert wird, sollte man aber nicht vorschnell als Hinweis darauf deuten, dass Gleichberechtigung im Streichquartett faktisch nicht funktioniert. Die humoristische Verkehrung der Verhältnisse ist eher ein weiteres Indiz für die normative Geltung eines Gleichberechtigungsideals im Streichquartett. Wie die hier genannten zum Teil gegenläufigen und bis heute verbreiteten Zuschreibungen aus der musikologischen Streichquartett-Theorie (Würde und Diskursivität) und dem lebensweltlich-literarischen Streichquartett-Mythos (Hierarchie und Streit) verdeutlichen, sind die Gleichberechtigungsmaxime und der Anspruch auf eine individuelle musikalische Verwirklichung in Form eines stummen Appells durchaus allgegenwärtig und daher Teil der musikalischen Sozialisation professioneller Streichquartettmusiker.

Das Spannungsverhältnis zwischen Individuum und Gruppe ist aber nicht nur als Hintergrundwissen in den Köpfen präsent, sondern stellt auch eine ganz praktische Herausforderung im Interaktionsprozess dar. Ebenso ist die besondere Intensität der Beziehungen unter den Musikern nicht nur eine Projektion von außen. Streichquartettmusiker verbringen viel Zeit miteinander in Proben und auf gemeinsamen Konzerttourneen, und das intensive Kennenlernen bis in die kleinste Gestik und Mimik hinein (Davidson und Good 2002, S. 190) bestimmt den Arbeitszusammenhang. Helga de la Motte-Haber weist darauf hin, dass ,die Schwierigkeit der gemeinsamen Aufgabenbewältigung [...] das Streichquartett zu einem klassischen Studienfall für Führung und Abstimmung“ machen (2007, S. 266). Der nächste Abschnitt gibt daher eine Bestandsaufnahme jener Studien, die die Interaktionen zwischen den Musikern empirisch näher untersucht haben.

\section{Streichquartett und Ensemblemusik als Studienfall}

Das Streichquartett wurde insbesondere im Hinblick auf seine Gruppendynamik zum Gegenstand wissenschaftlicher Untersuchungen verschiedener Disziplinen. In allen nachfolgend kurz vorgestellten Studien bildet die Probeninteraktion der Musiker zumindest einen Teil der Datenbasis. Helga de la Motte-Haber betont zwar in ihrem Beitrag zu sozialen Interaktionen in Musikgruppen in dem Band Musiksoziologie (2007, S. 260 ff.), das Streichquartett sei ein klassischer Studienfall für Führung und Abstimmung, verweist jedoch nur auf zwei musikpsychologische Studien, die sich dem Streichquartett widmen (Davidson und Good 2002; King 2006). Das kann als Indiz dafür gewertet werden, dass die Untersuchung von Streichquartetten - und von Ensemblemusik im Allgemeinen - bislang kein genuin soziologisches Interessensgebiet ist. Auf der Grundlage des psycholinguistischen Konzeptes des ,grounding“ (Clark und Brennan 1991) haben Jane W. Davidson und James M. M. Good (2002) untersucht, welche soziokulturellen und sozioemotionalen Phänomene in den Diskussionen der Musiker verhandelt werden, wie der musikalische Stil der Komposition die Koordination der Musiker untereinander erschwert oder begünstigt und wie kleine Gesten und Blickkontakte zur wechselseitigen Abstimmung genutzt werden. 
Die Studie von Elaine King (2006) widmet sich auf der Basis von Befragungen und Beobachtungen der Musiker den Rollenverteilungen innerhalb eines Streichquartetts, eines Bläserquartetts und eines Saxophonquartetts. King konstatiert eine dauerhafte Festlegung der Rollen im Streichquartett, die sich vergleichsweise positiv auf das musikalische Vorankommen im Verlauf der Proben auswirke, wohingegen die Musiker im Saxophon- und im Bläserquartett Rollenwechsel vollzögen. Auch J. Keith Murnighan und Donald E. Conlon (1991) untersuchen die Gruppendynamik im Streichquartett, wobei sich ihr Interesse auf den Zusammenhang zwischen Erfolg und Gruppendynamik richtet. Die genannten Arbeiten bewegen sich im Themenfeld weiterer sozialpsychologischer Studien, die sich der Organisation von Führungsübernahme und Führungsabgabe widmen (vgl. Gilboa und Tal-Shmotkin 2010; Tovstiga et al. 2004). Jane Ginsborg und Elaine King (2012) präsentieren in ihrer Studie einen Vergleich zwischen den Interaktionen professioneller Musiker und den Interaktionen studentischer Musiker hinsichtlich der Anzahl der Redebeiträge, der musikalischen Aspekte, die in der Probe thematisiert werden, und der Art der Übungsstrategien.

Eine Untersuchung, die sich stärker mit den Charakteristika der Kommunikation im Streichquartett und weniger mit der Rollenproblematik befasst, ist die von Frederick A. Seddon und Michele Biasutti (2009). Die Autoren identifizieren mithilfe einer thematischen Analyse der Probengespräche in Streichquartetten drei Kommunikationsmodi, die jeweils in verbaler und nonverbaler Form auftreten: Direktion, Kooperation und Kollaboration. Auch Amanda Bayleys (2012) Studie widmet sich der Art und Weise des Kommunizierens im Ensemble. Sie untersucht in ihrer ethnografischen Fallstudie zur Interaktion zwischen einem Komponisten und einem professionellen Streichquartett deren Probengespräche im Hinblick auf die Art der dort verwendeten Sprache. Dabei stellt sie unter anderem die Verwendung einer metaphorischen Sprache fest - ein Befund, der sich auch in der hier durchgeführten Untersuchung zeigen wird.

Konversationsanalytische Studien zur Kommunikation im Streichquartett sind hingegen vergleichsweise selten. Emanuel A. Schegloff, einer der Hauptvertreter der Konversationsanalyse, erläuterte in einem Interview eine allerdings noch unkonkrete Forschungsidee zum Streichquartett: „,The initial motivating idea was to examine several distinct orders of interactivity which supply the infrastructure for making music together: the embodied interactive conduct of the playing itself, the interaction at rehearsal through which the playing is developed, and the interaction written into the score by the composer" (Prevignano und Thibault 2003, S. 15f.). Eine erste Umsetzung dieser Idee findet sich in den konversationsanalytischen Studien des Anthropologen John B. Haviland (2007, 2011), die sich der Frage der Koordination im Streichquartett und in anderen Ensembleformaten widmen. Haviland konstatiert eine multimodale Koordination der Musiker, die aus dem komplexen Zusammenspiel der unterschiedlichen Ausdrucksmöglichkeiten von Sprache, Gesten, Gesang, Spiel und Mimik erwächst. Dem Prozess der musikalischen Koordination geht auch Daniela Veronesi (2014) in ihrer konversationsanalytischen Untersuchung zur Organisation von Korrekturen in der Interaktion zwischen Dirigent und Musikern nach. Sie arbeitet heraus, welche verbalen und nonverbalen Mittel zur Koordination genutzt werden, wenn die Beteiligten nicht auf eine Notation zurückgreifen können. Der Analyse von Korrektursequenzen widmet sich auch Peter Weeks in seinen Arbeiten (1996, 
2002). Mit Blick auf die Frage, wie Korrekturen und Anweisungen eines Dirigenten an ein Orchester kommuniziert werden, unterscheidet Weeks zwischen zwei kommunikativen Formaten: ,,verbal expressions“ und ,illustrative expressions“. Unter ersteren versteht er Spielanweisungen an die Musiker in wortsprachlicher Form. Unter letzteren fasst er alle Ausdrucksformen zusammen, die ohne die Verwendung von Worten die gewünschten Versionen zu spielender Stellen in der Musik veranschaulichen: dies kann durch Gesang geschehen, durch den bestimmte musikalische Eigenschaften markiert werden (z.B. Rhythmus, Tempo oder Tonhöhe), aber auch durch körperliche Ausdrucksformen wie das Klatschen erfolgen (Weeks 1996, S. 262 f.). Auch die Studie von Katherine Parton untersucht Orchester und die interaktive Wissensvermittlung durch Dirigenten. Parton stellt fest (2014, S. 416), dass Dirigenten ihre Bewertungen in der Kommunikation als subjektiv markieren, sodass andere Perspektiven möglich bleiben. Inwieweit die hier vorgelegte eigene Studie für diese Befunde weitere Evidenzen liefert, wird im Fazit ausführlicher diskutiert werden. An dieser Stelle bleibt zunächst festzuhalten, dass eine systematische Untersuchung von Bewertungsinteraktionen in Streichquartett-Probengesprächen noch aussteht. Im folgenden Abschnitt wird näher auf die Frage eingegangen, warum die ethnomethodologische Konversationsanalyse für die Erforschung der Bewertungskommunikation im Streichquartett eine geeignete Forschungsperspektive bietet.

\section{Zum ethnomethodologischen Blick auf Bewertungsprozesse im Streichquartett}

Die Bewertungsforschung in der Soziologie hat sich in jüngster Zeit vor allem mit dem Prozess des ,gatekeeping“ befasst. Dabei geraten Selektionsprozesse in unterschiedlichen sozialen Kontexten in den Blick, wie beispielsweise die Selektion von Beiträgen für Publikationen im wissenschaftlichen und literarischen Feld (Merriman 2015; Hirschauer 2006, 2015; Lamont 2009), von Literatur durch das Kritikerwesen (Chong 2011), von Kunst durch Kuratoren im Museum (Acord 2010), von Sexualpartnern (Green 2011) oder auch von Gästen durch die Türsteher eines exklusiven Nachtclubs (Rivera 2010). Bewertungsprozesse werden in diesen Studien vor allem im Hinblick auf die Legitimierung bestimmter Praktiken untersucht, die zur Anerkennung, Aufnahme oder zum Ausschluss von Akteuren bzw. deren Leistungen führen. Die Arbeiten knüpfen somit an Pierre Bourdieus (1982) Distinktionsansatz an, der auf positiv (oder negativ) auszeichnende soziale Wahrnehmungs- und Bewertungsakte abstellt.

Für die vorliegende Studie zur Bewertungskommunikation im Streichquartett ist jedoch nicht der Distinktionsaspekt, sondern der Integrationsaspekt von Bewertungen von Interesse. Ein Streichquartett ist kein kompetitiver, sondern ein kooperativer Arbeitszusammenhang (Motte-Haber 2007, S. 267). Die wechselseitigen Bewertungen des Spiels im Streichquartett unterliegen keiner Wettbewerbslogik, da sie nicht darauf abzielen, einzelnen Quartettmitgliedern einen ihren jeweiligen Leistungen entsprechenden Rang im hierarchischen Gefüge zuzuweisen. Die Kooperationsverpflichtung wird sogar eher unterlaufen, wenn einzelne Spieler ihre Virtuosität zu ausgiebig zur Schau stellen (Loft 2003, S. 179). Die Selektion von Leistungen 
im Streichquartett bezieht sich demnach nicht auf die Akteure, die ihre Interpretationsvorstellungen hervorbringen, sondern auf die Interpretationsvorstellungen selbst. Soll die Kooperation unter den vier Musikern fortbestehen, muss nach einer Bewertung auch derjenige mit der am wenigsten von den anderen geteilten Idee einer Interpretation weiterhin in der Gruppe integriert bleiben.

Eine Vorannahme für diese Studie ist daher, dass die Interaktionsteilnehmer im Streichquartett bei ihrer Bewertungskommunikation „Techniken der Imagepflege“ im Sinne von Erving Goffmans Konzept der Interaktionsrituale (1986) anwenden, um die Integration der Mitglieder durch ein Gleichgewicht in der Kommunikation zu gewährleisten. Dieses ,rituelle Gleichgewicht“ wird laut Goffman gewahrt, indem die Interaktionsteilnehmer darauf achten, das eigene Image - das Selbstbild - und das der anderen nicht zu gefährden. Der Vermeidungsprozess und der korrektive Prozess sind für Goffman die zwei grundlegenden Techniken der Imagepflege. Zum Vermeidungsprozess zählt er die kommunikative Vorbeugung, die Nichtbeachtung und die Verheimlichung von Imagegefährdungen (ebd., S. 21 ff.). Der korrektive Prozess reagiert hingegen auf einen imageschädigenden Zwischenfall, der sich nicht vermeiden ließ. Das beschädigte Image und das rituelle Gleichgewicht wird in diesem Fall öffentlich durch eine sogenannte Ausgleichshandlung wiederhergestellt (ebd., S. 25).

Im Anschluss an dieses Imagekonzept lassen sich Bewertungen nicht nur als Selektionsprozesse fassen, sondern als ,,alltägliche evaluative Praxis“ in der Kommunikation (Hirschauer 2006, S. 406), im Zuge derer die Angemessenheit von Äußerungen bewertet und sanktioniert wird. In diesem Horizont bewegt sich auch die Forschung zur kommunikativen Hervorbringung von moralischen Urteilen (vgl. Bergmann und Luckmann 1999). Insbesondere wurden negative Sanktionen in Alltagsgesprächen konversationsanalytisch erforscht wie das Sich-Beklagen (Christmann 1999), die Beschwerde und der Vorwurf (Günther 1999a, 1999b). Im Unterschied zur Alltagskommunikation handelt es sich bei der Interaktion im Streichquartett jedoch um eine aufgabenbezogene Kommunikation professioneller Musiker. Die hier gegebenenfalls auftretenden negativen Bewertungen sind also institutionell gerahmt, insofern sie ein fester Bestandteil von Probengesprächen und somit Teil der professionalisierten Kommunikation sind. Die Probleme einer möglichen Kränkung und Imageverletzung durch Bewertungen bleiben dennoch bestehen. Wie werden also im Streichquartett negative Bewertungen kommunikativ verhandelt, ohne dass das Ensemblemitglied, dessen Spiel negativ bewertet wurde, aus der professionellen Kooperation ausgeschlossen wird und sein „Gesicht“ einbüßt?

Für die empirische Bearbeitung dieser Frage eignet sich ein ethnomethodologischer Zugang. Die Ethnomethodologie, deren Entwicklung, so Heinz-Günter Vester (2010, S. 37), ,ohne die Arbeiten von Erving Goffman [...] schwer vorstellbar [ist]“, untersucht die konstitutiven Mechanismen, mittels derer Kommunikation strukturell organisiert wird, und widmet sich der Frage, ,,wie Menschen in interaktiven Prozessen soziale Wirklichkeit herstellen“ (Flick 2016, S. 86). Es geht also nicht in erster Linie darum herauszufinden, an welche Regeln sich die Akteure halten, sondern darum, welche kommunikativen Methoden sie nutzen, um einander anzuzeigen, inwiefern sie regelkonform handeln, oder anders ausgedrückt, was sie ,wechselseitig aufeinander bezogen tun [...], um diese Regeln auch faktisch einzuhalten oder sie zu überschreiten“ (Kleemann et al. 2013, S. 36). Als methodische Grundlage der 
nachfolgenden Untersuchung dient die ethnomethodologische Konversationsanalyse (KA). Bei diesem systematisierten Verfahren (Ten Have 1999, S. 48; Flick 2016, S. $422 \mathrm{ff}$.) werden natürliche Interaktionen auf Basis transkribierter Aufzeichnungen sequenzanalytisch analysiert (Kleemann et al. 2013; Bergmann 2013). Jörg Bergmann, der die Konversationsanalyse im deutschsprachigen Raum weiterentwickelt hat, erläutert deren Anliegen wie folgt: ,Die KA geht davon aus, dass in allen Formen von [...] Kommunikation die Handelnden damit beschäftigt sind, die Situation und den Kontext ihres Handelns zu analysieren, die Äußerungen ihrer Handlungspartner zu interpretieren, die situative Angemessenheit, Verständlichkeit und Wirksamkeit ihrer eigenen Äußerungen herzustellen und das eigene Tun mit dem Tun der anderen zu koordinieren“ (ebd., S. 525). Die Analyse der Verwendung sprachlicher Mittel bei der tatsächlich vollzogenen Bewertungspraxis im Streichquartett legt frei, wie Inhalte strukturiert werden und wie die Relevanz und Legitimation von Bewertungen kommunikativ so hergestellt werden, dass die Streichquartettmitglieder in das Kooperationsgeschehen integriert bleiben.

Bei der Auswahl der Sequenzen für die Analyse wurden zwei typische Gesprächssituationen berücksichtigt: Zum einen ist dies, in Anlehnung an die Idee der Reparaturorganisation in Gesprächen (Schegloff 1992), die Intervention, zum anderen der durch einzelne Interaktionsteilnehmer eingeleitete Gesprächsthemenwechsel, durch den das gesamte Probengespräch in einzelne Episoden mit ihren jeweiligen Eröffnungs- und Beendigungssequenzen unterteilt wird (zur Gesprächseröffnung vgl. ebenfalls Schegloff 1972; zum Gesprächsende Button 1987; Böhringer 2011). Die Intervention - die Unterbrechung des Spiels durch einzelne Mitglieder - ist für die Bewertungskommunikation insofern interessant, als hier die (negative) Bewertung des Spiels den Ausschlag für den Abbruch des Spiels gibt. Sie dient der Erarbeitung einer Interpretation, während sie zugleich durch das mit der Kritik einhergehende Kränkungspotenzial die Aufrechterhaltung der kommunikativen Prozesse gefährdet. Wie das Datenmaterial zeigt, kommt es im Zuge der Diskussion im Anschluss an eine Intervention oft zu weiteren Bewertungen über den ursprünglichen Anlass der Intervention hinaus. Deshalb ist auch der Themenwechsel der Kommunikation, der innerhalb einer Diskussion nach einer Intervention gegebenenfalls eine neue Bewertung markiert, von einem analytischen Interesse. In der Untersuchung wurden somit gezielt Interventions- und Themenwechsel-Sequenzen innerhalb von Diskussionen ausgewählt, analysiert und miteinander verglichen. Die nachfolgend vorgestellten Ergebnisse basieren auf der Analyse zahlreicher Sequenzen, von denen aufgrund des gegebenen beschränkten Rahmens nur einige wenige exemplarische Sequenzen herausgegriffen werden können.

\section{Zur Auswahl des Falls und der Sequenzen}

Die Datenbasis der Studie besteht aus über zwölf Stunden Audio- und Videomaterial verschiedener Proben. Bei dem beobachteten Streichquartett handelt es sich um ein studentisches Quartett, das sich zum Zeitpunkt der Datenerhebung noch in der Ausbildung an einer deutschen Musikhochschule befand. Ein studentisches Streichquartett bringt den forschungspraktischen Vorteil mit sich, dass es relativ leicht zugäng- 
lich ist. Davidson und Good nennen einen weiteren Aspekt, der angesichts der hier verfolgten Fragestellung für die Wahl eines studentischen Streichquartetts spricht. Musiker lernen erst im Laufe einiger Jahre, die Gestik und Mimik ihrer Mitspieler zu entschlüsseln (Davidson und Good 2002, S. 190). Im Umkehrschluss heißt das, dass sich Musiker, die noch nicht lange gemeinsam in einem Streichquartett spielen, stärker auf der verbalen Ebene verständigen, da sie sich noch nicht wortlos verstehen. Das ist für eine Untersuchung der verbalen Bewertungskommunikation natürlich eine unverzichtbare Voraussetzung.

Aus dem umfangreichen Datenmaterial wurde zunächst eine Interventionssequenz $\{\mathrm{S} 1\}$ herausgegriffen, anhand derer sich viele jener zentralen Befunde, die im Datenmaterial ausfindig gemacht wurden, veranschaulichen lassen. Um die Evidenz der Befunde und die Plausibilität der Interpretationen zu verdeutlichen, werden nachfolgend weitere acht Sequenzen $\{S 2\}-\{S 9\}$ aus dem erhobenen Datenmaterial herangezogen. Diese Sequenzen wurden im Zuge des Forschungsprozesses ursprünglich unabhängig einer ebenso ausführlichen Analyse wie Sequenz $\{$ S1 $\}$ unterzogen. Im beschränkten Rahmen eines Artikels kann dies aber nur partiell dargestellt werden. Den einzelnen Analyseabschnitten wurden kursiv formatierte Überschriften gegeben, welche die Interpretationen in einer Formulierung zusammenfassen, die die Art und Weise (,wie?“) und den Zweck (,,wozu?") der jeweiligen konversationsanalytisch herauspräparierten kommunikativen Form kenntlich machen sollen.

\section{Analyse von Interventions- und Themenwechsel-Sequenzen}

Die Transkription der Sequenzen basiert auf den Transkriptionskonventionen des Gesprächsanalytischen Transkriptionssystem 2 (GAT2) nach Selting et al. (2009, S. $391 \mathrm{ff}$.) und erfolgt daher generell in Kleinschreibung, da die Großbuchstaben zur Notation von Akzenten benötigt werden. In den Sequenzen wurden folgende Zeichen und Schreibweisen verwendet:

Sequenzielle Struktur/Verlaufsstruktur:

$\left[\begin{array}{l}\text { [ ] } \\ {[}\end{array}\right]$

$=$

Pausen:

(.)

$(-)$

$(--)$

$(---)$

$(0.5)$
Überlappungen und Simultansprechen

schneller, unmittelbarer Anschluss neuer Sprecherbeiträge oder Segmente (latching)

Mikropause, geschätzt, bis ca. 0,2 Sek. Dauer kurze geschätzte Pause von ca. 0,2-0,5 Sek. Dauer mittlere geschätzte Pause von ca. 0,5-0,8 Sek. Dauer längere geschätzte Pause von ca. 0,8-1,0 Sek. Dauer gemessene Pausen von ca. 0,5 Sek. Dauer (Angabe mit einer Stelle hinter dem Punkt) 
Sonstige segmentale Konventionen:

und_äh
ähm
$:$
$::$
$::$ :

Sonstige Konventionen:

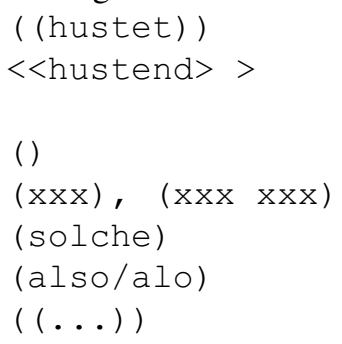

Akzentuierung:
akZENT
ak! ZENT!

\author{
Verschleifungen innerhalb von Einheiten \\ Verzögerungssignale, sogenannte ,gefüllte Pausen“ \\ Dehnung, Längung, um ca. 0,2-0,5 Sek. \\ Dehnung, Längung, um ca. 0,5-0,8 Sek. \\ Dehnung, Längung, um ca. 0,8-1,0 Sek.
}

para- und außersprachliche Handlungen und Ereignisse sprachbegleitende para- und außersprachliche Handlungen und Ereignisse mit Reichweite unverständliche Passage ohne weitere Angaben ein bzw. zwei unverständliche Silben vermuteter Wortlaut mögliche Alternativen Auslassung im Transkript

Tonhöhenbewegung am Ende von Intonationsphrasen:

$\begin{array}{ll}\text { ? } & \begin{array}{l}\text { hoch steigend } \\ \text { mittel steigend }\end{array} \\ - & \text { gleichbleibend } \\ ; & \text { mittel fallend } \\ \text { - } & \text { tief fallend }\end{array}$

Lautstärke- und Sprechgeschwindigkeitsveränderungen, mit Reichweite:

$\langle\langle\mathrm{acc}\rangle\rangle \quad$ accelerando, schneller werdend

\section{$\mathrm{Zu}$ Wort kommen:}

V1: Musiker an der ersten Geige

V2: Musiker an der zweiten Geige

B: Musiker an der Viola (Bratsche)

C: Musiker am Violoncello (Cello) ${ }^{3}$

Die nachfolgend präsentierten Sequenzen sind einer Probe entnommen, die in einem Seminarraum der Musikhochschule stattfand, an der die Musiker zum Zeitpunkt der Datenerhebung studierten. Im Anschluss an das erstmalige Durchspielen des Musikstücks spielte das Quartett das Stück von vorne an und arbeitete sich Stück für Stück

\footnotetext{
${ }^{3}$ Die Vereinheitlichung der Geschlechter erfolgt zum einen aus Gründen der Anonymisierung, denn eine Zuordnung der Geschlechter der Personen zu den jeweils von ihnen gespielten Instrumenten würde die Identifikation des Streichquartetts ermöglichen. Zum anderen richtet sich das Forschungsinteresse nicht auf die geschlechtsspezifische Bewertungskommunikation.
} 
in der Komposition voran. Wann immer eine Stelle aus Sicht eines Quartettmitglieds besprochen werden musste, wurde das Spiel unterbrochen und bewertet, was zumeist auch zu einer Wiederholung der betreffenden Stelle führte.

$\{\mathrm{S} 1\}$
$\{1\} 01$
((Quartett probt Stelle ab Takt 16))
$\{1\} 02 \mathrm{~V} 1:$
$[<<$ spielt eine Solostelle in Takt 23> $>$ ]
\{1\}03 B: [<<blickt in eigene Noten> eine frage noch $\mathrm{zu}]$ diesem->
$\{1\} 04$ C: ja: .
\{1\}05 B: ((spielt Takt 22 der eigenen stimme vor))
\{1\}06 B: ((blickt zu V2)) ich würd ! NICH! schlagen.
\{1\}07 B: ich würd alles (.) MÖglichst an der saite spielen;
$\{1\} 08$ V2: m_hm,
\{1\}09 B: es_es sieht so n_bisschen- (2.0)
\{1\}10 B: ( (macht mit der rechten Hand, den Bogen dabei in der typischen Geigen-/Bratschen- spieler-Haltung mit der rechten Hand haltend, eine von Augen- bis Brusthöhe fallende Geste))
\{1\}11 C: ((steht auf und schaut in Bs Noten))

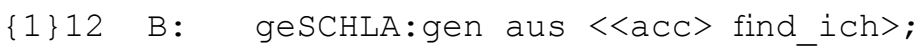
$\{1\} 13$ B: ((spielt)) es sieht so n_bisschen- ( (spielt)) so: aus.
\{1\}14 V1: [( (nimmt Partitur vom Boden))]
\{1\} 15 V2: [ja.
\{1\}16 V1: ((blickt in Partitur))

\section{\{1\}03: Diffuse Ansprache zur Vermeidung von Schuldzuweisungen}

Die Intervention in das Spiel erfolgt durch B. Während V1 eine Solostelle spielt, V2, $\mathrm{B}$ und $\mathrm{C}$ also eine vom Notentext vorgegebene Pause haben, kündigt $\mathrm{B}$ in $\{1\} 03$ mit „eine Frage noch zu diesem“ eine Frage an, wodurch er durchblicken lässt, dass ihn die Spielweise hier irritiert. Er gibt keinen Hinweis darauf, wessen Spielweise die Irritation auslöst - es könnte auch die eigene sein. Bs Frageabsicht ist nicht an bestimmte Mitspieler adressiert, da B in $\{1\} 03$ in die eigenen Noten blickt, und bleibt somit diffus. Diese Phase der Vorbereitung auf eine Bewertung gibt keinen Hinweis darauf, wessen Spiel im Folgenden bewertet wird, kommt also ohne ,offensive Anklage" einer am Missklang schuldigen Person aus.

Die kommunikative Form der diffusen Ansprache wird nicht nur in Vorbereitung auf eine Bewertung der Spielweise einer bestimmten Person genutzt. Es finden sich zahlreiche Sequenzen, in denen konsequent vermieden wird, eine konkrete Person gezielt anzusprechen, wie die folgende Sequenz $\{$ S2 $\}$ exemplarisch veranschaulicht. 
$\{\mathrm{S} 2\}$

$\{2\} 01$ B: also zuMINdest diesen TON.

\{2\}02 C: doch aber EIgentlich SCHON (.) gePLANT.

\{2\}03 B: jaja, ich habs auch geHÖRT <<acc> da_hast->

\{2\}04 C: m_mh, ja.

$\{2\} 05 \quad(---)$

\{2\}06 C: habt ihr AUCH sechsunddreißich punkte?

$\{2\} 07 \mathrm{~B}: \mathrm{ja}$;

$\{2\} 08 \quad(3.0)$

$\{2\} 09 \mathrm{V1}: \quad[j a-]$

\{2\}10 C: [dann] sollten wir die vielleicht mehr mit !SCHWUNG! DURCHnehmen als mit- () (beugt sich $\mathrm{zu}$ Bs Noten))

$\{2\} 11 \quad((\ldots))$

\{2\}12 V1: aber ACHtel MÜssen wir schon halten.

\{2\}13 C: ja_JA;

\{2\}14 aber man kann die auch mit SCHWUNG nehmen einfach; wenn man ( (spielt stelle vor)) is ANders als- ((spielt Stelle vor))

$\mathrm{C}$ beginnt in $\{2\} 06$ ein neues Thema nach einer Einigung zu einem anderen Diskussionspunkt in $\{2\} 04$. Interessant daran sind im soeben besprochenen Zusammenhang die Plural-Formulierungen ,habt ihr" in $\{2\} 06$ und ,sollten wir“ in $\{2\} 10$, die in $\{2\} 12$ von V1 durch ,müssen wir“ fortgesetzt werden, wobei die Diffusität der Ansprache (,man kann“) auch in $\{2\} 14$ von C aufrechterhalten wird. In beiden Sequenzen wird die Kritik bzw. der Einstieg in die Kritik als sachorientiert und nicht personenorientiert gekennzeichnet: Im Fokus steht nicht die Person, die etwas falsch gemacht hat, sondern ein zu behebender Sachverhalt. Die Kommunikation wird damit nicht nur als aufgabengebundene gerahmt, sondern es wird zugleich auch einer Schuldzuweisung vorgebeugt. Bereits vor dem Themenwechsel findet sich in $\{2\} 03$ die Vermeidung einer direkten Adressierung. Hier hätte B sein Satzfragment „da hast“ um ein „Du“ sowie um ein Prädikat und Objekt zu einem vollständigen Satz ergänzen können. Stattdessen bricht er seinen Satz jedoch vor dem „Du“ ab, was die Lesart der Präferenz einer diffusen Ansprache bestätigt. Doch die Äußerung in der ersten Sequenz in $\{1\} 03$ (,eine Frage noch zu diesem“) lässt noch eine weitere Interpretation $\mathrm{zu}$.

\section{\{1\}03: Einleitende Frage(absicht) zum Abbau hierarchischer Distanz und zur Öffnung des Diskussionsraums}

Durch die Ankündigung einer Frage (,eine Frage noch zu diesem“) gibt B zu verstehen, dass ihm ein Sachverhalt nicht klar ist. Er deutet somit Unsicherheit in Bezug auf die eigene Spielweise an. Durch seine Frageabsicht demonstriert B nicht nur seine Irritation, sondern auch seine scheinbare Ahnungslosigkeit, wodurch B den Befragten einerseits die Möglichkeit gibt, sich als Experten zu erweisen, andererseits eine belehrende Haltung vermeidet. Viel auffälliger als die hier beobachtete Frageabsicht sind im Datenmaterial jedoch tatsächliche Fragen, mit denen Bewertungen oder eine 
Kritik eingeleitet werden. Ein Blick zurück auf Sequenz $\{$ S2 $\}$ zeigt, dass C seinen Themensprung auch mit einer Frage markiert: ,habt ihr auch sechsunddreißich Punkte?“ (\{2\}06). Eine analoge Sequenz findet sich an anderer Stelle:

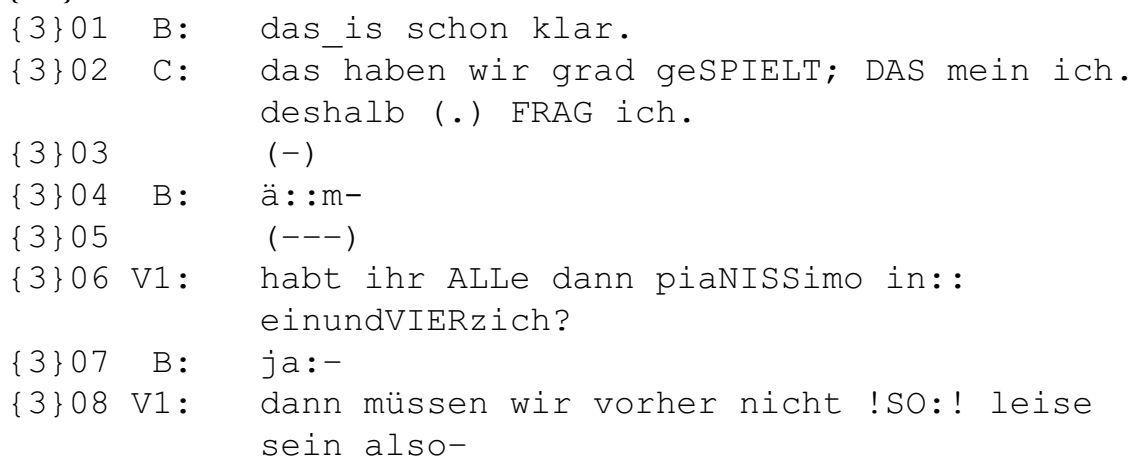

Hier ist es V1, der nach dem Platzhalter ,äm“ in \{3\}04, mit dem B die kurze Pause nach Beendigung der vorherigen Diskussion überbrückt, einen Themensprung mit der Frage „habt ihr alle dann pianissimo in einundvierzich?“ (\{3\}06) einleitet und damit zugleich die anderen auf seine Kritik vorbereitet. Durch die Frage wird signalisiert, dass die Situation der anderen bei der eigenen Argumentation Berücksichtigung findet. Es kommt aber auch vor, dass die Kritik ohne Vorbereitung auskommt, dafür aber selbst schon als direkt adressierte Frage formuliert ist:

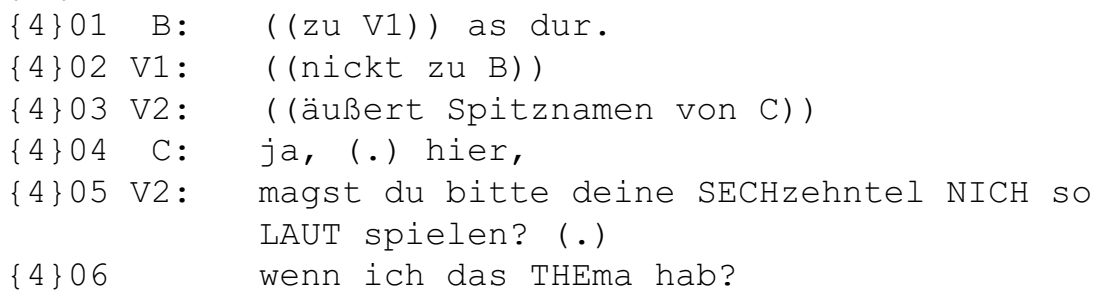

Auch diese Sequenz zeigt einen Themensprung, der in $\{4\} 03$ durch V2 eingeleitet wird. Durch die in $\{4\} 05$ als Frage gestellte Kritik ,magst du bitte deine Sechzehntel nich so laut spielen?“ wird das Gegenüber dazu eingeladen, sich zu der Kritik zu äußern. Durch die Formulierung „,magst du“ zeigt V2 an, dass ihm die Präferenzen von $\mathrm{C}$ wichtig sind, sodass die Kritik trotz direkter Ansprache durch das „Du“ - die hier inhaltlich schwer zu vermeiden ist, da $C$ hier als einziger Sechzehntel spielt - entschärft wirkt. Der Kritisierte bekommt auch durch die Frage die Möglichkeit, Gegenargumente einzubringen, was im Fall der im Imperativ stehenden reinen Anweisung „Spiel nicht so laut, wenn ich das Thema habe!“ nicht gegeben wäre. Die Kombination der Bewertung mit einer Frage ist, wie die Sequenzen $\{\mathrm{S} 1\},\{\mathrm{S} 3\}$ und $\{\mathrm{S} 4\}$ beispielhaft zeigen, eine weitere kommunikative Form, mittels derer hierarchische Distanz abgebaut wird, da dadurch die eigene Urteilshoheit aufgegeben wird. 
Indem die Bewertung durch die Fragestellung diskutabel erscheint, wird zugleich der Diskussionsraum geöffnet.

\section{\{1\}04: Zustimmung zwecks Legitimierung einer Intervention als Verweis auf deren Begründungsbedürftigkeit}

Interessant ist, dass $\mathrm{C}$ sich in $\{1\} 04$ trotz diffuser Ansprache durch B veranlasst sieht, auf dessen Redebeitrag zu reagieren. Mit seinem „Ja“ signalisiert B Zustimmung. Faktisch ist dies keine zustimmende Antwort auf eine gestellte Frage, sondern C stimmt hier einer Frageabsicht zu. Er gibt auf diese Weise zu verstehen, dass er eine Frage zu der angezeigten Stelle für gerechtfertigt hält, legitimiert dadurch Bs Unterbrechung und ebnet den Weg für Bs weitere Argumentation. Dies nimmt B die Aufgabe ein Stück weit ab, die Unterbrechung selbst zu begründen, womit C B einerseits entgegenkommt, andererseits durchaus auch die Begründungsbedürftigkeit von Interventionen anzeigt, was ein Hinweis auf deren Kränkungspotenzial ist.

\section{\{1\}05: Klangbeispiel zur Darstellung einer Klangvorstellung}

Im Anschluss spielt B in $\{1\} 05$ die Stelle im Stück vor, auf die sich seine Frageabsicht bezieht. Es wäre auch möglich gewesen, die Stelle durch die Nennung der Taktzahl zu kennzeichnen. Sie vorzuspielen ist jedoch eindeutiger, da es sich bei der Stelle nicht um den ganzen Takt, sondern um eine Tonfolge innerhalb des Takts handelt. Somit dient die Ergänzung der sprachlichen Darstellung durch das Spiel zugleich der Vereinfachung und Präzisierung. Darüber hinaus leistet das Vorspielen der Stelle noch einen weiteren Dienst, indem es als akustisches Beispiel und Vorbild dient. B bringt hier, ohne es verbal zu erwähnen, seinen Vorschlag für die richtige Spielweise ein.

\section{\{1\}06: Perspektivenübernahme zum Abbau hierarchischer Distanz}

Bis hierher ist in Sequenz $\{\mathrm{S} 1\}$ durch B weder eine Bewertung oder Kritik, noch ein verbal geäußerter Verbesserungsvorschlag vorgebracht worden. In \{1\}06 formuliert B nun einen Verbesserungsvorschlag, den er an V2 richtet: ,ich würd nich schlagen“. Was mit dem Wort „schlagen“ gemeint sein könnte, wird an späterer Stelle ausgeführt. Wichtig ist zunächst die Feststellung, dass hier Bs diffus adressierte Frageabsicht aus $\{1\} 03$ durch den hergestellten Blickkontakt in einen Ratschlag an V2 mündet. Dies verstärkt den Eindruck, dass die Vermeidung einer Schuldzuweisung und der Abbau der hierarchischen Distanz in $\{1\} 03$ die Funktion haben, das abzufedern, was nun kommt, und V2 darauf vorzubereiten: die eigentliche Kritik bzw. Bewertung. Interessant ist, wie B seinen Verbesserungsvorschlag formuliert: ,ich würd nich schlagen“ steht im Konditional bzw. im Konjunktiv II (,würde“ plus Infinitiv), was ihn deutlich von einer im Imperativ stehenden, hierarchische Distanz herstellenden Aufforderung abgrenzt wie z. B. ,Schlag nicht!“” oder „Hör auf zu schlagen!“‘. Stattdessen zeigt B durch seine Formulierung an, dass er sich in die Lage von V2 versetzt, und argumentiert aus dessen imaginierter Perspektive heraus. Der Satz lässt sich zu ,ich würde an deiner Stelle nicht schlagen“ ergänzen. Anstelle eines Befehls wird somit Empathie angezeigt. 
\{1\}06: Kennzeichnung der Bedingtheit des Vorschlags zur Öffnung des

\section{Diskussionsraums}

Nicht nur ein Perspektivwechsel und Empathie werden durch diese Formulierung angezeigt. Der Konditional kennzeichnet etwas Irreales, nur Vorgestelltes, dessen Eintreten an eine nicht unbedingt gegebene Bedingung geknüpft ist. Durch seine Verwendung hier wird daher auch angezeigt, dass der Ratschlag an eine Bedingung geknüpft ist und daher, sollte die Bedingung nicht erfüllt sein, ein Rückzug in eine der anderen Möglichkeiten der Interpretation offen steht. Bs Satz lässt sich somit auch ergänzen durch, ,ich würde nicht schlagen, wenn ich du wäre - da ich aber nicht du bin, kann es auch anders sein“. Die Knüpfung der Aussage an eine Bedingung öffnet den Diskussionsraum, denn die Möglichkeit eines Gegenarguments bleibt gewahrt und der Ratschlag von B wird nicht als unumstößliche Wahrheit präsentiert.

Die kommunikative Öffnung des Diskussionsraums wurde bereits an anderer Stelle angesprochen, nämlich im Hinblick auf eine die Bewertung begleitende Fragestellung, die die Kritik als diskutabel erscheinen lässt. Das Kennzeichnen der Bedingtheit der Kritik durch die Verwendung des Konditionals ist eine weitere kommunikative Form der Ermöglichung dieser Öffnung. Was diese kommunikativen Formen verbindet, ist, dass durch sie das Gegenüber nicht vor ,,vollendete Tatsachen“ gestellt wird. Vielmehr wird der Ausschluss von Endgültigkeit kommuniziert, es kann der Bewertung widersprochen werden. Ein weiterer Befund im Datenmaterial, der zu diesem Analyseergebnis führt, ist das Signalisieren der Subjektivität von Kritik:

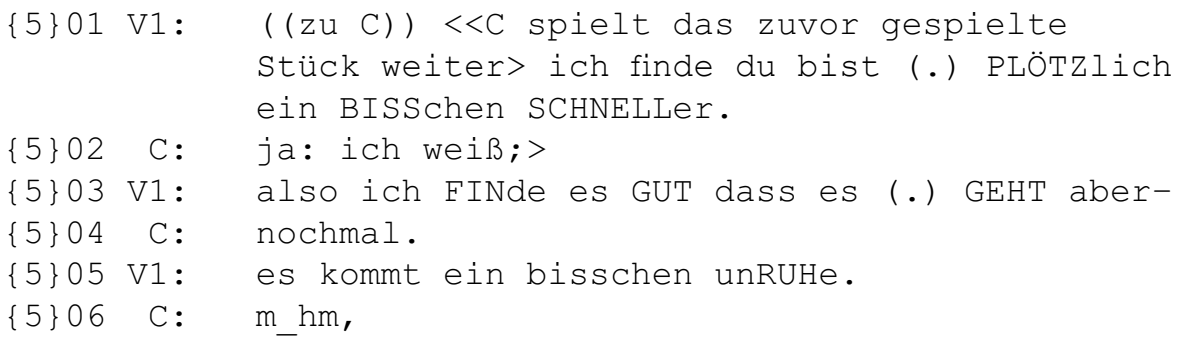

Auch die Formulierung ,,ich finde“ kann als Einladung zum Gespräch verstanden werden: Der Kritisierende öffnet die Kommunikation für die Möglichkeit des Widerspruchs, indem er aus seiner subjektiven Perspektive heraus argumentiert und dadurch mit seiner Aussage keinen Anspruch auf Allgemeingültigkeit erhebt. Was man selbst findet, müssen andere noch lange nicht so finden. Was an dieser Sequenz auBerdem ins Auge fällt, ist die Kombination der Kritik mit einem Lob in \{5\}03, die besänftigend wirkt. Die folgende Sequenz $\{$ S6 $\}$, die eine Gegenargumentation von C enthält, dessen Spiel zuvor von V2 als zu laut bewertet wurde, verweist ebenfalls auf das Kennzeichnen von Subjektivität: 
$\{$ S6 $\}$
\{6\}01 C: ich finde geneRELL eher, $=$
$\{6\} 02 \mathrm{C}: \quad$ dass (.) (das/dass) ALLes was (.) ähm was wir hier so spielen auf der WEIchen sEIte is. $=$
\{6\}03 =ich fin ich fänds schön wenn der Klang
n_bisschen konzenTRIERter wär;
\{6\}04 weil dann wirds DURCHsichtich;
$\{6\} 05 \mathrm{VI}: \quad \mathrm{m}_{-} \mathrm{hm}$,
$\{6\} 06 \quad(\overline{-})$
$\{6\} 07$ B: ja das_gut;
\{6\}08 C: also wenn ich zu LAUT spiele dann dann dann spiel ich LEIser;
\{6\}09 aber ich finde trotzdem dass es mit !KERN! (-) [SEIN sollte. ]
$\{6\} 10$ B: [(das auch gut.)]
\{6\}11 V1: [jajajaJA. ] DAS schon.
\{6\}12 C: <<leise> das würd ich auch UNgern (.) ANders spielen.>
\{6\}13 B: !NE:! aber vielleicht ist eher dass du WEIßt, dass_s_da $(x x x) \cdot\left[<<g e l a c h t>a ̈ \_h a ̈->\right]$
$\{6\} 14 \mathrm{C}: \quad$ [jaJA (.) das schon]

\section{(.) SCHON klar.}

C ändert das im Indikativ stehende Wort ,finde“ aus \{6\}01 zu „fänds“ in \{6\}03. Die Subjektivierung seiner Bewertung wird nun mit dem Konditional verbunden. Auch im weiteren Verlauf kennzeichnet C die Bedingtheit seiner Aussage durch ,wenn-“, bzw. ,wenn-dann“-Formulierungen (vgl. \{6\}03, \{6\}08). Wenngleich C auf inhaltlicher Ebene auf seinem Standpunkt beharrt (,,das würd ich auch ungern anders spielen“" in \{6\}12), so erzeugt er durch die Wahl seiner Grammatik die Möglichkeit der Erosion seines Standpunkts: Hier hätte $\mathrm{C}$ beispielsweise deutlicher durch die Wendung „Das will ich nicht anders spielen!“ insistieren können.

Bislang kamen durch die Analyse und Deutung der Äußerung in $\{1\} 06$ aus der ersten Sequenz nun also schon zwei Befunde zum Vorschein: die Perspektivenübernahme zum Abbau hierarchischer Distanz und, wie auch unter Hinzuziehung der Sequenzen $\{$ S5 $\}$ und $\{$ S6 $\}$ weiter verdeutlich wurde, die Kennzeichnung der Bedingtheit eines Vorschlags zwecks Öffnung des Diskussionsraums. Die weitere Analyse dieser Stelle weist zunächst auf einen Punkt hin, der bereits thematisiert wurde:

\section{\{1\}06: Vermeidung des „Du“ zur Umgehung der Schuldzuweisung}

Die Kritik am Spiel von V2, die B durch die Aussage ,,ich würd nich schlagen“ einbringt, ist eher implizit als explizit formuliert und erfährt somit eine Abschwächung. B hätte auch ,es klingt nicht gut, wie du hier schlägst“", sagen können. B vermeidet jedoch auch in der direkten Ansprache an V2 das ,Du“ und markiert somit V2 nicht als Schuldigen am Missklang. Dies lässt sich als Analogie zu dem lesen, was bereits in 
Bezug auf die diffuse Ansprache gesagt wurde. Wie oben angekündigt, geht die folgende Analyse näher auf die Einordnung des Wortes „,schlagen“ in diesem Satz ein.

\section{\{1\}06: Verwendung metaphorischer Sprache zur Beschreibung einer}

Klangvorstellung

Das Verb „schlagen“ taucht während der Proben mehrmals auf, was die Vermutung nahe legt, dass dieser Ausdruck unter den Musikern gängig ist. Aus dem Videomaterial (siehe Beschreibung der Geste in $\{1\} 10)$ geht hervor, dass damit eine ausholende Armbewegung mit dem Bogen gemeint ist, auf die eine vergleichsweise schnelle, schwungvolle und unter erhöhtem Kraftaufwand vollzogene Beförderung des Bogens auf die Saite folgt. Eine derart detaillierte Beschreibung dieser Bewegung leistet das Wort „,schlagen“ jedoch nicht. Es impliziert vielmehr eine technische Spielanweisung, indem es einen komplexen Bewegungsablauf in einem Wort verdichtet, statt diesen explizit zu beschreiben. Wenngleich es an dieser Stelle auch nicht aus der Sequenz hervorgeht, so ist doch aufgrund der Häufigkeit der Verwendung des Wortes „schlagen“ im gesamten Material davon auszugehen, dass es sich um eine sprachliche Konvention unter Musikern handelt. In der musikalischen Fachsprache findet sich eine Reihe von Ausdrücken, die dem außermusikalischen Sprachgebrauch entlehnt sind. Nicht selten sind diese Worte in ihrem ursprünglichen Sinn mit Emotionen oder Bildern assoziiert, beispielsweise die aus dem Italienischen stammenden Angaben zum Tempo wie allegro (fröhlich, lebhaft, leicht) oder auch adagio (langsam, ruhig), das sich aus ad und agio (Behagen, Bequemlichkeit, Muße) zusammensetzt. Die Metaphorik in den Konventionen der musikalischen Fachsprache, wie sich auch im Wort „schlagen“ zeigt, kann - gerade weil sie an das Vorstellungsvermögen der Musiker appelliert - möglicherweise präziser zum Ausdruck bringen, welche Spieltechnik gemeint ist, als dies eine konkrete Beschreibung von Bewegungsabläufen zu leisten vermag. Dennoch bleiben auch solche metaphorischen Bezeichnungen deutungsoffen. Dies weist auf eine spezifische Herausforderung in der Kommunikation unter Musikern hin, nämlich die der Übersetzung einer Klangvorstellung in die verbale Sprache. Das betrifft auch die inhaltliche Begründung einer Bewertung des Spiels. Die Analyse des Materials hat ergeben, dass sich das Kommunizieren einer Klangvorstellung als schwierig erweist. Folgende Sequenz veranschaulicht das Ringen um Worte:

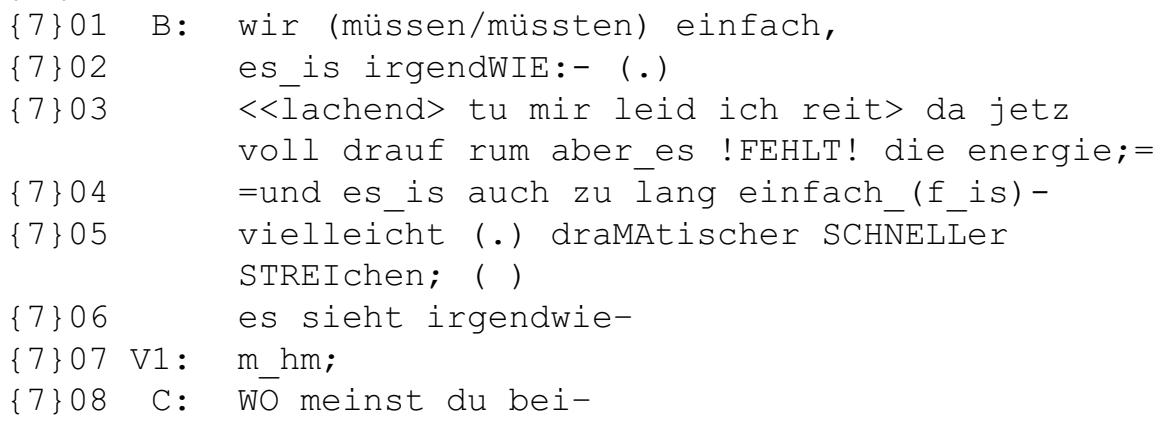




\begin{tabular}{|c|c|c|}
\hline 7$\} 09$ & B: & [ich find; \\
\hline 7$\} 10$ & $C:$ & [((spielt Takt 22 eigene stimme))] \\
\hline & B : & ja wenn die das zu ZWEIT haben; = \\
\hline & & $\begin{array}{l}\text { =ich find (.) da ! FEHLT! irgendwo kommt es } \\
\text { das erste mal so WIschi. }\end{array}$ \\
\hline & $C:$ & $\mathrm{ml}$ \\
\hline & V2: & $\mathrm{mh}$ \\
\hline , & B : & $\begin{array}{l}\text { da ist zuerst mal im FORte, und dann (---) } \\
\text { !FEHLT! mir einfach grade was. (-) [aber-] }\end{array}$ \\
\hline & & \\
\hline & & \\
\hline
\end{tabular}

Umschrieben wird der zu erzielende Klang mit den Worten „Energie“, „,dramatischer“, „schneller Streichen“; der gehörte und kritisierte Klang wird mit „wischi“ beschrieben. Abgesehen von der Wendung „schneller Streichen“, die eine konkrete technische Spielanweisung darstellt, appellieren die Redefiguren an ein Vorstellungsvermögen der Adressaten. Andere bereits angeführte Sequenzen unterstützen diesen Befund. So ist in $\{$ S6 $\}$ die Rede von der ,weichen Seite“ (\{6\}02) und einem Klang, der schöner wäre, wenn er „konzentrierter“ (\{6\}03) wäre, da er sonst drohe, ,,durchsichtich“ ( $\{6\} 04)$ zu werden. In $\{$ S5 $\}$ konstatiert V1 „Unruhe“( $\{5\} 05)$ im Spiel von $\mathrm{C}$, und auch in den folgenden Sequenzen finden sich Worte, die zumeist in anderen Kontexten verwendet werden, als bei der Beschreibung von Klang.
$\{8\} 01$ V1:
( (zu v2)) ja. (-) du bist einfach zu LEIse.
$\{8\} 02$ B: also zu UNkonkret vielleicht.
$\{9\} 01$ V1:
$\{9\} 02 \mathrm{C}:$
naja (.) [es ist bei mir auch. ]
$\{9\} 03$ V1:
[wie hab ich DAS geschafft?]
ich denke die die KLANGfarbe ist einfach nicht RICHtig.
\{9\}04 V1: also es klingt als (.) THEma weil es so ganz (.) DICHT.

Der in der deutschen Sprache bestehende Mangel an Worten, die vornehmlich Klangbeschreibungen vorbehalten sind und für die eindeutige Bezeichnung von Klangformen und -mustern verwendet werden können (z.B. ,schrill“), macht die Klangbeschreibung - und damit die inhaltliche Begründung von Bewertungskriterien für musikalische Praktiken - zu einer kommunikativen Herausforderung. Das Datenmaterial zeigt, dass vor allem zwei Arten der Klangbeschreibung verwendet werden: Zum einen umfassen sie konkrete, mehr oder weniger detaillierte spieltechnische und bewegungsbezogene Anweisungen. Beispiele dafür in den untersuchten Sequenzen sind die Anweisungen „schneller Streichen“ (\{7\}05), also eine schnellere Bewegung mit dem Bogen auf der Saite, und ,an der Saite spielen“ (\{1\}07), womit gemeint ist, dass V2 beim Abheben des Bogens von der Saite nicht so weit ausholen, sondern 
den Bogen näher am Instrument lassen soll. Die zweite Art der Klangbeschreibung umfasst das Ausweichen auf Ausdrücke, die nicht-musikalischen Bedeutungskontexten entnommen sind. Listet man die Worte auf, mit denen die Musiker in den analysierten Probengesprächen versuchen, den gewünschten Klang zu erklären, zeigt sich, dass vielfach eine metaphorische oder stimmungsbeschreibende Sprache zum Einsatz kommt: „dramatischer“, „Energie“, „geschlagen“, „,durchsichtig“, „, unkonkret“, ,konzentrierter“, ,,weich“, „mit Kern“, ,dicht“, „,wischi“, „Unruhe“. Mit diesen Worten appellieren die bewertenden Musiker an das Vorstellungsvermögen ihres Gegenübers. Dadurch zeigen sie an, dass die Perspektive und die Gefühlswelt des Gegenübers in die eigene Argumentation integriert werden. Die bewertende Person zielt darauf $a b$, dass der Adressierte auf emotionaler Ebene versteht, warum seine Spielpraxis kritisiert wird, was über eine rein technische Spielanweisung hinausgeht. Da diese Worte außermusikalischen und emotional assoziierten Bedeutungskontexten entnommen sind und auf das individuelle Erleben und Wahrnehmen von Klängen zielen, entziehen sich ihre Sinngehalte einer Verallgemeinerung. Dies bleibt in der Bewertungskommunikation über Klangvorstellungen ein dauerhaftes Problem.

\section{\{1\}07 Kennzeichnung der Bedingtheit des Vorschlags zur Öffnung des Diskussionsraums}

In \{1\}07 findet sich erneut die Formulierung ,ich würd“. Die zusätzliche Anreicherung des Konditionals durch den Superlativ „,möglichst“ unterstreicht die Bedingtheit des Vorschlags: Nur wenn es möglich ist, würde B alles an der Saite spielen. Die Möglichkeit der Spielbarkeit ist die Bedingung, an die der Verbesserungsvorschlag geknüpft wird. Dadurch wird auch der Vorschlag-Charakter der Bewertung durch B unterstrichen: Die von B vorgebrachte Lösung zur Beseitigung der Irritation, die durch die Spielweise von V2 ausgelöst wird, wird von B als subjektiv gekennzeichnet. Gegenvorschläge werden dadurch nicht ausgeschlossen; die Bewertungspraxis erweist sich auch hier als ein potenziell offener Prozess.

\section{\{1\}08: Knappe Einverständnisbekundung zur Öffnung des Diskussionsraums}

V2 reagiert auf den Vorschlag von B in $\{1\} 08$ mit die Äußerung ,m-hm“. Die leicht ansteigende Tonhöhe gibt einen Hinweis darauf, dass V2 Bs Bewertung akzeptiert, nicht ablehnt. Solche knappen Zustimmungsäußerungen tauchen in den Sequenzen sehr häufig auf. V2 hätte seine Zustimmung auch offen artikulieren können, beispielsweise durch ,genau, gute Idee“ oder ,ja, du hast Recht“. Der Kritisierte thematisiert somit seine kritikwürdige Spielweise nicht weiter, wodurch die Abweichung seiner Spielweise zur erwünschten nicht weiter hervorgehoben wird. Dies legt einerseits die Interpretation nahe, dass Kritik als unangenehm wahrgenommen wird und der Schritt zur Kränkung nicht weit ist. Es lässt sich daraus andererseits aber auch schlussfolgern, dass bei mancher Einigung noch ein Restzweifel bestehen bleibt. Trotz einer formalen Einigung durch ,m-hm“ kann das Thema aufgrund des relativen Mangels an Verbindlichkeit dieser Einverständnisbekundung jederzeit wieder aufgegriffen werden. Dies kann als weiterer Hinweis auf eine Öffnung des Diskussionsraums durch die Ermöglichung einer Neubewertung gedeutet werden. Eine Neubewertung ist angesichts des Umstands, dass es sich um Probengespräche handelt, durchaus wahrscheinlich. 


\section{\{1\}09: Beschreibung der Visualität des Spiels zur Beschreibung einer}

\section{Klangvorstellung}

In $\{1\} 09$ setzt B seine Argumentation fort mit der Äußerung ,,es es sieht so $\mathrm{n}$ bisschen". Interessant ist, dass B auf das Aussehen von V2s Spielweise verweist und nicht auf die Qualität des Klangs selbst eingeht. Entweder er stört sich an der visuellen Ästhetik an sich oder er betrachtet das, was er gesehen hat, als Erklärung für das, was er gehört hat: Was geschlagen aussieht, klingt geschlagen, da die Bewegung Teil der Klangerzeugung ist. Ändert V2 das, was an seiner Spielweise visuell wahrnehmbar ist, ändert sich folglich auch der Klang. Das Ausweichen auf die visuelle Beschreibung des Gespielten zwecks Beschreibung einer Klangvorstellung kann als Hinweis darauf gedeutet werden, dass die bislang durch B gegebene Klangbeschreibung von ihm selbst als unzureichend empfunden wurde.

\section{\{1\}09: Vermeidung des „Du“ zur Umgehung der Schuldzuweisung}

Mit der Äußerung „es es sieht so n bisschen“ vermeidet B auch an dieser Stelle wieder das „Du“. Ebenso hätte B sagen können „du spielst ein bisschen“. Das Subjekt, von dem die Klangaktivität ausgeht, ist jedoch ein unbestimmtes „Es“. Somit wird auch hier der Urheber des Missklangs nicht als „Schuldiger“ benannt.

\section{\{1\}10: Gestische Ergänzung zur Beschreibung einer Klangvorstellung}

$B$ beendet den Satz in $\{1\} 09$ nicht, sondern pausiert seine Rede, um sie im unmittelbaren Anschluss mit einer Geste zu ergänzen. Die in $\{1\} 10$ beschriebene Geste fällt in die relativ lange Sprechpause von B. Da niemand anderes das Wort ergreift, ist die Sprechpause nicht als Beendigung des Redebeitrags zu deuten. Die Kommunikation von B setzt sich durch die Geste vielmehr fort, was die anderen dazu veranlasst, nicht das Wort zu ergreifen. Die Geste hat die Funktion zu erklären, was mit „schlagen“ gemeint ist: der Schlag des Bogens auf die Saiten der Violine. B hält seinen Bogen dabei weiterhin in der üblichen Spielhaltung und richtet den Bogen auch nicht anders aus. Er schlägt jedoch nicht auf die Saite, sondern in die Luft. Durch die Geste ergänzt er seine sprachliche Äußerung - möglicherweise, weil das Wort „schlagen“ allein mehrere Deutungsmöglichkeiten eröffnet, was wiederum darauf hinweist, dass der ausschließliche Rückgriff auf eine metaphorische Sprache zur Erläuterung der Spielweise unzulänglich wäre.

\section{\{1\}12: Bekräftigung der Subjektivität zur Öffnung des Diskussionsraums}

In $\{1\} 12$ setzt B seinen Satz im Anschluss an seine Geste mit der Äußerung ,geschlagen aus find ich" fort. Er wiederholt das Wort ,geschlagen“ unter starker Betonung und fügt den schnell gesprochenen Zusatz ,find ich“ an. Bs Aussage wäre auch ohne diesen Zusatz verständlich. Durch die dadurch signalisierte Subjektivierung seiner Aussage wird erneut das Gespräch für die Möglichkeit des Widerspruchs geöffnet.

\section{\{1\}13: Klangbeispiel zur Darstellung einer Klangvorstellung}

In $\{1\} 13$ spielt B die besprochene Stelle an, ohne dies im Vorfeld angekündigt zu haben. Dennoch ist die Deutung naheliegend, dass es sich hierbei nicht um ein Üben der Stelle handelt, sondern um ein Vorspielen, das der Unterstützung seiner Argumentation dient. Anschließend wiederholt B seinen Satz „es sieht so $\mathrm{n}$ bisschen“ aus $\{1\} 09$. 
Statt der Geste, die in $\{1\} 10$ auf $\{1\} 09$ folgt, spielt er nun die betreffende Stelle noch einmal vor und bekräftigt durch ein anschließendes betontes „So“, wie die Spielweise von V2 aussieht. Um die aus seiner Sicht kritikwürdige Spielweise von V2 zu charakterisieren, bedient sich B also einer metaphorischen Beschreibung (,geschlagen“), einer gestischen Imitation der kritisierten Bewegung und einer klanglichen Imitation der negativ bewerteten Spielweise von V2. Er charakterisiert den Klang in mehreren Anläufen, was die Interpretation stützt, dass jeder einzelne dieser Anläufe für sich als unzureichend für die Übermittlung einer Klangvorstellung erachtet wird.

\section{\{1\}14: Parallelaktivität von V1 und C während der Kommunikation zwischen B und V2}

Der Blick in die Partitur, den V1 in $\{1\} 14$ vornimmt, stellt eine Analogie zu Cs Aktivität in $\{1\} 11$ dar. V1 verortet hier seine Stimme in Relation zu den anderen Stimmen. Sowohl C als auch V1 greifen jedoch nicht in die Interaktion ein, die zwischen $\mathrm{B}$ und V2 stattfindet. Ein Grund hierfür könnte sein, dass es nicht zu einem Konflikt zwischen B und V2 kommt und es daher keiner Schlichtung durch einen Dritten bedarf.

\section{\{1\}15: Knappe Einverständnisbekundung zur Öffnung des Diskussionsraums}

In $\{1\} 15$ reagiert V2 mit einem fallend intonierten ,Ja“ und signalisiert zum einen Zustimmung, zum anderen, auch durch das Senken der Stimme, dass das Thema für ihn beendet ist. Die knappe Art der Zustimmung erinnert an das, was bereits anhand von V2s Äußerung in $\{1\} 08$ beobachtbar wurde: Das Thema kann aufgrund dieses relativen Mangels an Verbindlichkeit jederzeit wieder aufgegriffen werden.

\section{Diskussion und Fazit}

Die in dem Beitrag vorgelegte exemplarische Analyse der Interventionssequenz $\{\mathrm{S} 1\}$ unter Hinzuziehung weiterer Sequenzen aus dem Datenmaterial offenbart eine defensive Bewertungspraxis unter Streichquartettmusikern. Dies deckt sich mit dem von Goffman beschriebenen Vermeidungsprozess - einem der beiden grundlegenden kommunikativen „Techniken der Imagepflege“. Wie bereits erwähnt, umfasst der Vermeidungsprozess solche Handlungen, die der Vorbeugung, der Nichtbeachtung oder der Verheimlichung von Imagegefährdungen dienen, sodass die Interaktionsteilnehmer ihr Selbstbild und das der anderen wahren können (Goffman 1986, S. 21 ff.). Die Fallanalyse hat gezeigt, dass von den Musikern kränkende Formulierungen und persönliche Schuldzuweisungen sowie die kommunikative Herstellung hierarchischer Distanz vermieden werden. Es ist naheliegend, davon auszugehen, dass in einem Streichquartett der Wunsch nach dem Fortbestehen persönlicher Beziehungen den Austauschprozess bestimmt und wechselseitige Bewertungen somit milder ausfallen (zu einem gleichen Schluss, wenn auch für einen ganz anderen Personenkreis, kommt Rivera 2010).

Darüber hinaus legte die Analyse eine durch unterschiedliche kommunikative Formen erzeugte Öffnung und Offenhaltung des Diskussionsraums frei. Die Bewertungen werden von den Akteuren als subjektiv und diskutabel markiert (,,ich find“), und 
Formulierungen im Konditional (,,ich würd“) drücken die Bedingtheit von Bewertungen aus. In dieser Hinsicht zeigen sich Parallelen zu den Forschungsergebnissen von Katharine Partons Untersuchung von Interaktionen zwischen Dirigent und Orchester: „By marking his assessments as based on his own, personal (,I', ,to me') and subjective judgements, the Conductor is also allowing for the possibility of other perspectives on this rich and complex soundscape. Marking a judgement as perceptual rather than as factual allows, however slimly, for the potentiality of other valid perceptions." (Parton 2014, S. 416) Dazu passt die Beobachtung aus der vorliegenden Studie, dass das Akzeptieren von Bewertungen durch die Bewerteten in Form von eher knappen, wenig verbindlich erscheinenden Äußerungen der Zustimmung zum Ausdruck gebracht wird (,,ja“, ,,m-hm“), die die Fortführung einer offenen Diskussion im Bewertungsprozess ermöglichen: Man lässt sich kommunikativ „ein Hintertürchen offen“, die Verbindlichkeit in der Kommunikation der Musiker wird aufgeweicht. Dieses Ergebnis ist insofern erstaunlich, als hier nicht, wie bei den Vermeidungsprozessen in Interaktionsritualen, für das Selbstbild negative Sachverhalte vermieden werden, sondern etwas im Alltagsverständnis positiv Konnotiertes vermieden wird: Verbindlichkeit. Zu einem ähnlichen Befund sind J. Keith Murnighan und Donald E. Conlon gekommen, die als eine Strategie des Umgangs mit Meinungsverschiedenheiten im Streichquartett hinsichtlich einer Interpretation den Aufschub der Entscheidung ausgemacht haben. Diese sogenannte ,time-out“- oder ,,cooling-off“-Periode ermöglicht ein Wiederaufgreifen der Diskussion zu einem späteren Zeitpunkt (Murnighan und Conlon 1991, S. 177). Angesichts der besonderen Fallauswahl der vorliegenden Studie liegt die Deutung nahe, dass in einem studentischen Streichquartett der Grad an Unsicherheit in Bezug auf die angemessene Spielweise eines Musikstückes höher ist als bei einem Streichquartett, das schon seit Jahren miteinander musiziert, und deshalb die Interpretationsentscheidungen aufgrund dieser größeren Unsicherheit weniger verbindlich kommuniziert werden. Einen Anhaltspunkt für diese Vermutung bieten Jane Ginsborg und Elaine King, die den Interaktionsstil professioneller und studentischer Duos für Gesang und Klavier miteinander verglichen haben. Sie kommen zu dem Schluss: „Students were more likely than professionals to show solidarity and ask for orientation, while professionals were more likely to give and ask for opinions" (Ginsborg und King 2012, S. 148). Dass die Vermeidung verbindlicher Aussagen und Vorgaben jedoch ausschließlich ein Zeichen mangelnder professioneller Erfahrung ist, erscheint angesichts eines weiteren Befunds der Studie von Murnighan und Conlon (1991, S. 177) wenig plausibel: Die Autoren verweisen darauf, dass erfolgreiche professionelle Streichquartette strittige Interpretationsalternativen in verschiedenen Konzerten ausprobieren. Die Möglichkeit des Experimentierens mit unterschiedlichen Interpretationen wird einer verbindlichen Einigung auf eine einzige Interpretation vorgezogen. Auch ein Zitat aus einer Unterrichtssituation des von mir beobachteten Streichquartetts stützt die These, dass die beobachtete Offenheit bzw. das Fehlen von Verbindlichkeit kein eindeutiger Beleg für eine spezifisch studentische Unsicherheit ist. Ein lehrender Kammermusik-Professor antwortete auf die Frage nach der geeigneten Spielweise einer kniffeligen Stelle: „Ich will kein Rezept verraten. Ich weiß ja selber keins. “ Diese Form der Vermeidung unumstößlicher Sollaussagen und Bewertungen ist bemerkenswert: Bewertungen erhalten eher den 
Anstrich von Bewertungen unter Vorbehalt. Sie dienen der gemeinsamen Sondierung von Möglichkeiten, nicht der Erreichung bereits feststehender Ziele.

Für eine Soziologie, die sich der kommunikativen Bewertungspraxis widmet, stellt sich die weiterführende interessante Frage, inwiefern sich die in dieser Fallstudie beobachtete Offenheit der Bewertungskommunikation - die Bewertung unter Vorbehalt - auf andere Berufsgruppen übertragen lässt. Anzunehmen ist, dass die Verbindlichkeit in der Bewertungskommunikation zunimmt, wenn die Konsequenzen von Bewertungen weitreichend sind. ,Wo folgenreiche Entscheidungen zu treffen sind, finden sich regelmäßig hoch komplexe Verfahren der Kontingenzreduktion des Bewertens." (Hirschauer 2006, S. 407) Wie in Abschnitt 4 erwähnt, widmet sich die soziologische Bewertungsforschung insbesondere dem Selektionsprozess des für die bewerteten Akteure sehr folgenreichen ,gatekeeping“ (vgl. Merriman 2015; Hirschauer 2006, 2015; Lamont 2009; Chong 2011; Acord 2010; Rivera 2010). Eine Ausweitung der Bewertungsforschung auf Bereiche, in denen, wie hier untersucht, eher die Integration statt die Selektion der bewerteten Akteure im Mittelpunkt steht, könnte den Sinn für die Kontextabhängigkeit und Vielfalt von Bewertungssituationen schärfen. Ob die „Bewertung unter Vorbehalt“ eine für Musiker typische Form der Bewertungskommunikation ist oder dieses Phänomen auch in anderen Kontexten auftritt, bleibt durch weitere Untersuchungen zu klären.

In den Sequenzen zeigt sich auch, dass die sprachliche Übersetzung und Vermittlung einer Klangvorstellung eine kommunikative Herausforderung für alle Beteiligten darstellt. Es fällt einem Musiker schwer, eine Bewertung zu begründen und zu verteidigen, wenn es kein verlässliches intersubjektiv geteiltes sprachliches Instrumentarium für die Formulierung der Bewertungskriterien selbst gibt. Das Problem der Unübersetzbarkeit von Musik in Sprache bleibt ein grundlegendes, und die verschiedenen Wege, dieses Problem praktisch zu lösen - über eine metaphorische Sprache, eine gestische Imitation der Spielbewegung oder ein beispielhaftes Vorspielen und Vorsingen (vgl. hierzu auch Bayley 2012; Haviland 2007, 2011; Weeks 1996; Veronesi 2014) -, verstärken den Eindruck, dass von den Musikern jeder dieser Wege für sich genommen als nicht ausreichend empfunden wird.

Fällt es schon schwer, die eigene individuelle Klangvorstellung mit der zur Verfügung stehenden Sprache zu kommunizieren, so setzt die Synchronisation dieser individuellen Klangvorstellungen aller Musiker in Form einer von allen geteilten gemeinsamen Interpretation ein hohes Maß an Vertrautheit voraus, und zwar sowohl mit dem Gegenstand, über den kommuniziert wird, als auch mit den individuellen verbalen und nonverbalen Ausdrucksweisen der anderen Streichquartettmitglieder. Insbesondere wenn das normative Ideal einer Gleichberechtigung der Kooperationsmitglieder mit dem Anspruch einer Wahrung der musikalischen Individualität der Musiker einhergeht, ist die individuelle Klangvorstellung nicht mehr nur ein durch die musikalische Sozialisation vorgegebenes Faktum, das es im Zuge der Anpassung an die anderen Musiker zu unterdrücken gilt, sondern sie ist die Voraussetzung einer gelungenen Interpretation des Musikstückes. Abschließend bleibt also festzuhalten, dass sich der beobachtete geöffnete Diskussionsraum beim wechselseitigen Bewerten von musikalischen Leistungen im hierarchiefreien Raum nicht nur aus der Vermeidung von Kränkungen erklärt, sondern auch aus der Tatsache, dass zur Kommunikation der musikalischen Bewertungskriterien keine eindeutige Sprache zur Verfügung 
steht. Die Schwierigkeit, wenn nicht Unmöglichkeit, Klangvorstellungen eindeutig in sprachliche Äußerungen und Anweisungen zu übersetzen, führt dazu, dass es keinen feststehenden und endgültigen „Katalog“ von Bewertungskriterien für Musik geben kann. Die Bewertungskommunikation bleibt strukturell offen, weil die Bewertungskriterien selbst laufend neu verhandelt werden. Eine Soziologie der Bewertung könnte sich der Frage annehmen, inwieweit sich dieser Befund auf andere Kommunikationskontexte übertragen ließe, in denen Bewertungskriterien strittig sind.

Open access funding provided by Paris Lodron University of Salzburg.

Open Access Dieser Artikel wird unter der Creative Commons Namensnennung 4.0 International Lizenz (http://creativecommons.org/licenses/by/4.0/deed.de) veröffentlicht, welche die Nutzung, Vervielfältigung, Bearbeitung, Verbreitung und Wiedergabe in jeglichem Medium und Format erlaubt, sofern Sie den/ die ursprünglichen Autor(en) und die Quelle ordnungsgemäß nennen, einen Link zur Creative Commons Lizenz beifügen und angeben, ob Änderungen vorgenommen wurden.

\section{Literatur}

1. Acord, S. K. (2010). Beyond the head: The practical work of curating contemporary art. Qualitative Sociology, 33, 447-467.

2. Adorno, T. W. (2001). Zu einer Theorie der musikalischen Reproduktion. Frankfurt a. M.: Suhrkamp.

3. Aulich, B., \& Heimeran, E. (1936). Das stillvergnügte Streichquartett. München: Heimeran.

4. Bäuerle, L. (Hrsg.). (1997). Bratschen-Witze. Zürich: Atlantis-Musikbuch-Verlag.

5. Bayley, A. (2011). Ethnographic research into contemporary string quartet rehearsal. Ethnomusicology Forum, 20, 385-411.

6. Bergmann, J. (2013). Konversationsanalyse. In U. Flick, E. v. Kardoff \& I. Steinke (Hrsg.), Qualitative Forschung. Ein Handbuch (S. 524-537). Reinbek: Rowohlt.

7. Bergmann, J., \& Luckmann, T. (Hrsg.). (1999). Kommunikative Konstruktion von Moral (2 Bde. Aufl.). Opladen: Westdeutscher Verlag.

8. Böhringer, D. (2011). Verabredungen am Gesprächsende - dargestellt am Beispiel von Gesprächen im Jobcenter. Forum Qualitative Sozialforschung/Forum: Qualitative Social Research. http://nbn-resolving.de/urn:nbn:de:0114-fqs110348. Zugegriffen: Juni 2016.

9. Bourdieu, P. (1982). Die feinen Unterschiede. Kritik der gesellschaftlichen Urteilskraft. Frankfurt a. M.: Suhrkamp.

10. Brügge, J. (2004). Wolfgang Rihms Streichquartette. Aspekte zu Analyse, Ästhetik und Gattungsgeschichte des modernen Streichquartetts. Saarbrücken: Pfau.

11. Button, G. (1987). Moving out of closings. In G. Button \& R. L. John (Hrsg.), Talk and social organization (S. 101-151). Clevedon: Multilingual Matters.

12. Chong, P. (2011). Reading differences: How race and ethnicity function as tools for critical appraisal. Poetics, 39, 64-84.

13. Christmann, G. B. (1999). „Sich-Beklagen“ in Ökologiegruppen. In J. Bergmann \& T. Luckmann (Hrsg.), Struktur und Dynamik der Formen moralischer Kommunikation Kommunikative Konstruktion von Moral, (Bd. 1, S. 151-173). Opladen: Westdeutscher Verlag.

14. Clark, H. H., \& Brennan, S. E. (1991). Grounding in communication. In L. B. Resnick, J. M. Levine \& S. D. Teasley (Hrsg.), Perspectives on socially shared cognition (S. 127-149). Washington, DC: American Psychological Association.

15. Davidson, J. W., \& Good, J. M. M. (2002). Social and musical co-ordination between members of a string quartet: An exploratory study. Psychology of Music, 30, 186-201.

16. v. Ehrmann, A. (1903). Das Streichquartett. Phantasien eines Musikers. Linz: Österreichische Verlagsanstalt.

17. Fink, G. W. (1837). Quartett. In Encyclopädie der gesammten musikalischen Wissenschaften oder Universal-Lexicon der Tonkunst (Bd. 5, S. 590-592). Stuttgart: Köhler.

18. Finscher, L. (1965). Streichquartett. In F. Blume (Hrsg.), Die Musik in Geschichte und Gegenwart (Sp. 1925-1927). Kassel: Bärenreiter-Verlag. 
19. Finscher, L. (1974). Studien zur Geschichte des Streichquartetts I - Die Entstehung des klassischen Streichquartetts. Von den Vorformen zur Grundlegung durch Joseph Haydn. Kassel: Bärenreiter.

20. Flick, U. (2016). Qualitative Sozialforschung. Eine Einführung. Reinbek: Rowohlt.

21. Gilboa, A., \& Tal-Shmotkin, M. (2010). String quartets as self-managed teams: An interdisciplinary perspective. Psychology of Music, 40, 19-41.

22. Ginsborg, J., \& King, E. (2012). Rehearsal talk: Familiarity and expertise in singer-pianist duos. Musicae Scientae, 16, 48-167.

23. Goethe, J. W. (1976). Goethes Briefe und Briefe an Goethe. Bd. 4. München: C. H. Beck.

24. Goffman, E. (1986). Interaktionsrituale. Über Verhalten in direkter Kommunikation. Frankfurt a. M.: Suhrkamp.

25. Green, A. I. (2011). Playing the (sexual) field: The interactional basis of systems of sexual stratification. Social Psychology Quarterly, 74, 244-266.

26. Günther, S. (1999a). Beschwerdeerzählungen als narrative Hyperbeln. In J. Bergmann \& Luckmann (Hrsg.), Struktur und Dynamik der Formen moralischer Kommunikation Kommunikative Konstruktion von Moral, (Bd. 1, S. 174-205). Opladen: Westdeutscher Verlag.

27. Günther, S. (1999b). Vorwürfe in der Alltagskommunikation. In J. Bergmann \& T. Luckmann (Hrsg.), Struktur und Dynamik der Formen moralischer Kommunikation Kommunikative Konstruktion von Moral, (Bd. 1, S. 206-241). Opladen: Westdeutscher Verlag.

28. Haviland, J. B. (2007). Master speakers, master gestures: A string quartet master class. In S. D. Duncan, J. Cassell \& E. T. Levy (Hrsg.), Gesture studies: Gesture and the dynamic dimension of language. Essays in honor of David McNeill (S. 147-172). Amsterdam: John Benjamins Publishing Company.

29. Haviland, J. B. (2011). Musical spaces. In C. Goodwin, C. Le Baron \& J. Streeck (Hrsg.), Multimodality and human activity: Research on human behavior, action, and communication (S. 289-304). Cambridge: Cambridge University Press.

30. Hirschauer, S. (2006). Wie geht Bewerten? - Zu einer anderen Evaluationsforschung. In U. Flick (Hrsg.), Qualitative Evaluationsforschung (S. 405-423). Reinbek: Rowohlt.

31. Hirschauer, S. (2015). How editors decide. Oral communication in journal peer review. Human Studies, 38, 37-55.

32. Hub, U. (1997). Die Beleidigten. Frankfurt a. M.: Verlag der Autoren.

33. King, E. C. (2006). The roles of student musicians in quartet rehearsals. Psychology of Music, 34, 262-282.

34. Kleemann, F., Krähnke, U., \& Matuschek, I. (2013). Interpretative Sozialforschung. Eine Einführung in die Praxis des Interpretierens. Wiesbaden: Springer VS.

35. Lamont, M. (2009). How professors think: Inside the curious world of academic judgment. Cambridge, MA: Harvard University Press.

36. Loft, A. (2003). How to succeed in an ensemble. Reflections on a life in chamber music. Portland: Amadeus Press.

37. Merriman, B. (2015). The editorial meeting at a little magazine: An ethnography of group judgement. Journal of Contemporary Ethnography, 1-24. doi:10.1177/0891241615615918.

38. de la Motte-Haber, H. (2007). Soziale Interaktionen von Musikgruppen. In H. de la Motte-Haber \& H. Neuhoff (Hrsg.), Musiksoziologie (S. 260-275). Laaber-Verlag: Laaber.

39. Murnighan, K., \& Conlon, D. E. (1991). The dynamics of intense work groups: A study of British string quartets. Administrative Science Quarterly, 36, 165-186.

40. Parton, K. (2014). Epistemic stance in orchestral interaction. Social Semiotics, 24, 402-419.

41. Prevignano, C. L., \& Thibault, P. J. (2003). Discussing Conversation Analysis: The work of Emanuel A. Schegloff. Amsterdam: John Benjamins Publishing Company.

42. Rivera, L. (2010). Status distinction in interaction: Social selection and exclusion at an elite nightclub. Qualitative Sociology, 33, 229-255.

43. Salmen, W. (1969). Haus- und Kammermusik. Privates Musizieren im gesellschaftlichen Wandel zwischen 1600 und 1900. Leipzig: Deutscher Verlag für Musik.

44. Schegloff, E. A. (1972). Sequencing in conversational openings. In J. J. Gumperz \& D. Hymes (Hrsg.), Directions in Sociolinguistics (S. 346-380). New York: Holt, Rinehart \& Winston.

45. Schegloff, E. A. (1992). Repair after next turn: The last structurally provided defense of intersubjectivity in conversation. American Journal of Sociology, 97, 1295-1345.

46. Schütte, W. (1991). Scherzkommunikation unter Orchestermusikern. Interaktionsformen in einer Berufswelt. Tübingen: Narr. 
47. Schütz, A. (1972). Gemeinsam Musizieren. In A. Schütz (Hrsg.), Gesammelte Aufsätze II: Studien zur soziologischen Theorie (S. 129-150). Den Haag: Martinus Nijhoff.

48. Seddon, F., \& Biasutti, M. (2009). Modes of communication between members of a string quartet. Small Group Research, 40, 115-137.

49. Selting, M., et al. (2009). Gesprächsanalytisches Transkriptionssystem 2 (GAT2). Gesprächsforschung - Online-Zeitschrift zur verbalen Interaktion, 10, 353-402. http://www.gespraechsforschung-ozs.de/heft2009/px-gat2.pdf.

50. Simmenauer, S. (2008). Muss es sein? Leben im Quartett. Berlin: Berenberg.

51. Ten Have, P. (1999). Doing conversation analysis: A practical guide. London: Sage.

52. Tovstiga, G., Odenthal, S., \& Goerner, S. (2004). Sense making and learning in complex organisations: The string quartet revisited. International Journal of Management Concepts and Philosophy, $1,215-231$.

53. Veronesi, D. (2014). Correction sequences and semiotic resources in ensemble music workshops: The case of Conduction. Social Semiotics, 24, 468-494.

54. Vester, H.-G. (2010). Kompendium der Soziologie III: Neuere soziologische Theorien. Wiesbaden: VS Verlag für Sozialwissenschaften.

55. Vicentino, N. (1555). L'antica musica ridotta alla moderna prattica. Rom: Antonio Barre.

56. Weeks, P. (1996). A rehearsal of a Beethoven passage: An analysis of correction talk. Research on Language and Social Interaction, 29, 247-290.

57. Weeks, P. (2002). Performative error-correction: A problem for ethnomethodological description. Human Studies, 25, 359-385.

Désirée Wilke geb. 1984. Studium der Soziologie an der Goethe-Universität Frankfurt am Main, derzeit Universitätsassistentin und Dissertantin an der Paris Lodron Universität Salzburg in der Abteilung für Soziologie und Kulturwissenschaft. Forschungsschwerpunkte: Kunst- und Musiksoziologie. 